\title{
FURTHER INEQUALITIES FOR THE (GENERALIZED) WILLS FUNCTIONAL
}

\author{
DAVID ALONSO-GUTIÉRREZ, MARÍA A. HERNÁNDEZ CIFRE, \\ AND JESÚS YEPES NICOLÁS
}

\begin{abstract}
The Wills functional $\mathcal{W}(K)$ of a convex body $K$, defined as the sum of its intrinsic volumes $\mathrm{V}_{i}(K)$, turns out to have many interesting applications and properties. In this paper we make profit of the fact that it can be represented as the integral of a log-concave function, which, furthermore, is the Asplund product of other two log-concave functions, and obtain new properties of the Wills functional (indeed, we will work in a more general setting). Among others, we get upper bounds for $\mathcal{W}(K)$ in terms of the volume of $K$, as well as Brunn-Minkowski and Rogers-Shephard type inequalities for this functional. We also show that the cube of edge-length 2 maximizes $\mathcal{W}(K)$ among all 0 -symmetric convex bodies in John position, and we reprove the well-known McMullen inequality $\mathcal{W}(K) \leq e^{\mathrm{V}_{1}(K)}$ using a different approach.
\end{abstract}

\section{INTRODUCTION, NOTATION AND MAIN RESULTS}

Let $\mathcal{K}^{n}$ be the set of all convex bodies, i.e., non-empty compact convex sets, in the $n$-dimensional Euclidean space $\mathbb{R}^{n}$. Let $\langle\cdot, \cdot\rangle$ and $|\cdot|$ be the standard inner product and the Euclidean norm in $\mathbb{R}^{n}$. For $K \in \mathcal{K}^{n}$ containing the origin in its interior, $|x|_{K}=\min \{\lambda \geq 0: x \in \lambda K\}$ will denote the well-known Minkowski functional of $K$, which, in the case when $K$ is 0 -symmetric (i.e., $K=-K$ ), defines a norm whose unit ball is $K$.

We represent by $B_{2}^{n}$ the $n$-dimensional Euclidean (closed) unit ball and by $\mathbb{S}^{n-1}$ its boundary. The volume of a measurable set $M \subset \mathbb{R}^{n}$, i.e., its $n$-dimensional Lebesgue measure, is denoted by $\operatorname{vol}(M)$ (or $\operatorname{vol}_{n}(M)$ if the distinction of the dimension is useful). In particular, we write $\kappa_{n}=\operatorname{vol}\left(B_{2}^{n}\right)$, which takes the value

$$
\kappa_{n}=\frac{\pi^{n / 2}}{\Gamma\left(\frac{n}{2}+1\right)},
$$

where $\Gamma(s)=\int_{0}^{\infty} t^{s-1} e^{-t} \mathrm{~d} t$, for $s>0$, represents the Gamma function.

2000 Mathematics Subject Classification. Primary 52A20, 52A39; Secondary 26B25, $52 \mathrm{~A} 40$.

Key words and phrases. Wills functional, intrinsic volumes, log-concave functions, projection function, Asplund product, Brunn-Minkowski type inequalities, Rogers-Shephard type inequalities, John position, McMullen's inequality.

The work is partially supported by MICINN/FEDER projects MTM2016-77710-P and PGC2018-097046-B-I00, by DGA E26_17R and by "Programa de Ayudas a Grupos de Excelencia de la Región de Murcia", Fundación Séneca, 19901/GERM/15. 
The Grassmannian of $k$-dimensional linear subspaces of $\mathbb{R}^{n}$ is denoted by $\mathrm{G}(n, k)$, and for $H \in \mathrm{G}(n, k)$, the orthogonal projection of $M$ onto $H$ is denoted by $P_{H} M$, whereas the orthogonal complement of $H$ is represented by $H^{\perp}$. Moreover, for $v \in \mathbb{S}^{n-1}$ and $t \in \mathbb{R}$, we write $H_{v, t}=\left\{x \in \mathbb{R}^{n}\right.$ : $\langle x, v\rangle=t\}$. With int $M$, bd $M$ and conv $M$ we denote the interior, boundary and convex hull of $M$, respectively. Finally, as usual in the literature, $\operatorname{SL}(n)$ stands for the subgroup of volume-preserving and orientation-preserving linear transformations.

For convex bodies $K, E \in \mathcal{K}^{n}$ and a non-negative real number $\lambda$, the wellknown Steiner formula states that the volume of the Minkowski sum $K+\lambda E$ can be expressed as a polynomial of degree (at most) $n$ in the parameter $\lambda$,

$$
\operatorname{vol}(K+\lambda E)=\sum_{i=0}^{n}\left(\begin{array}{l}
n \\
i
\end{array}\right) \mathrm{W}_{i}(K ; E) \lambda^{i} ;
$$

here, $\mathrm{W}_{i}(K ; E)$ are the relative quermassintegrals of $K$ with respect to $E$, and they are a special case of the more general defined mixed volumes (see e.g. [25, Section 5.1]). In particular, $\mathrm{W}_{0}(K ; E)=\operatorname{vol}(K)$ and $\mathrm{W}_{n}(K ; E)=$ $\operatorname{vol}(E)$. As usual in the literature, we shorten $\mathrm{W}_{i}\left(K ; B_{2}^{n}\right)=\mathrm{W}_{i}(K)$. In this case we observe that if $K \in \mathcal{K}^{n}$ has dimension $\operatorname{dim} K=k$, then we can obtain the $i$-th quermassintegral $\mathrm{W}_{i}(K)$ in $\mathbb{R}^{n}$, but also its $i$-th quermassintegral computed in the $k$-dimensional affine subspace where $K$ is contained (identified with $\mathbb{R}^{k}$ ), which we denote by $\mathrm{W}_{i}^{(k)}(K), i=0, \ldots, k$. These two numbers do not coincide; indeed we have (see e.g. [24, Property 3.1])

$$
\mathrm{W}_{i}^{(k)}(K)=\frac{\left(\begin{array}{c}
n \\
n-k+i
\end{array}\right)}{\left(\begin{array}{c}
k \\
i
\end{array}\right)} \frac{\kappa_{i}}{\kappa_{n-k+i}} \mathrm{~W}_{n-k+i}(K), \quad i=0, \ldots, k,
$$

with $\mathrm{W}_{i}(K)=0$ for $i=0, \ldots, n-k-1$. In order to avoid the issue that quermassintegrals depend on the space where the convex body is embedded, McMullen [21] defined the intrinsic volumes of a convex body $K \in \mathcal{K}^{n}$ as

$$
\mathrm{V}_{i}(K)=\frac{\left(\begin{array}{c}
n \\
i
\end{array}\right)}{\kappa_{n-i}} \mathrm{~W}_{n-i}(K), \quad i=0, \ldots, n .
$$

The particular case $i=1$ defines another well-known functional associated to a convex body: the mean width $\mathrm{b}(K)$ of $K$. More precisely,

$$
\mathrm{V}_{1}(K)=\frac{n}{\kappa_{n-1}} \mathrm{~W}_{n-1}(K)=\frac{n \kappa_{n}}{2 \kappa_{n-1}} \mathrm{~b}(K),
$$

and so it can be expressed in terms of the support function $h_{K}$ of $K$ as

$$
\mathrm{V}_{1}(K)=\frac{1}{\kappa_{n-1}} \int_{\mathbb{S}^{n-1}} h_{K}(u) \mathrm{d} u
$$

([25, page 50 and (5.57)]); here $\mathrm{d} u$ stands for the Lebesgue measure on $\mathbb{S}^{n-1}$. We recall that the support function of $K, h_{K}(u)=\max \{\langle z, u\rangle: z \in K\}$ for $u \in \mathbb{S}^{n-1}$, is a convex function that uniquely determines the convex body (see e.g. [25, Section 1.7]). 
In 1973 (see [31]) Wills introduced and studied the functional

$$
\mathcal{W}(K)=\sum_{i=0}^{n}\left(\begin{array}{l}
n \\
i
\end{array}\right) \frac{\mathrm{W}_{i}(K)}{\kappa_{i}}=\sum_{i=0}^{n} \mathrm{~V}_{i}(K)
$$

because of its possible relation with the so-called lattice-point enumerator $G(K)=\#\left(K \cap \mathbb{Z}^{n}\right)$, and conjectured that $\mathcal{W}(K)$ bounded by above $G(K)$. Although Hadwiger [16] showed that Wills' conjecture was wrong (see also $[9])$, the Wills functional turned out to have several interesting applications, e.g., in Discrete Geometry, where there exist nice relations of this functional with the so-called successive minima of a convex body [33], or in deriving exponential moment inequalities for Gaussian random processes [26]; see also [27, 28, 30]. It has also been studied in other probabilistic context, [29], and the behavior of its roots has been analyzed when it is seen as a formal polynomial in a complex variable, [17, 18, 32].

The Wills functional exhibits many nice and engaging properties. For instance, observe that $\mathcal{W}(K)$ depends only on the convex body, but not on the dimension of the embedding space. In the next theorem we enumerate some properties of $\mathcal{W}(K)$.

Theorem A. Let $H \in \mathrm{G}(n, k)$ and let $K, E \in \mathcal{K}^{n}$.

i) Hadwiger $[15,(2.3)]$ : if $K \subset H$ and $E \subset H^{\perp}$, then

$$
\mathcal{W}(K) \mathcal{W}(E)=\mathcal{W}(K+E) .
$$

ii) Hadwiger $[15,(2.4)]$ : for all $v \in \mathbb{S}^{n-1}$ and any $r \geq 0$,

$$
\mathcal{W}(K) \geq \frac{\mathcal{W}\left(K \cap H_{v, 0}\right)+\mathcal{W}\left(K \cap H_{v, r}\right)}{2}+\int_{0}^{r} \mathcal{W}\left(K \cap H_{v, t}\right) \mathrm{d} t .
$$

iii) Wills $[32,(4.4)]$ : the $i$-th derivative of $\mathcal{W}\left(-\lambda B_{2}^{n}\right)$ satisfies

$$
\frac{d^{i} \mathcal{W}\left(-\lambda B_{2}^{n}\right)}{d \lambda^{i}}=\frac{n ! \kappa_{n}}{i ! \kappa_{i}} \mathcal{W}\left(-\lambda B_{2}^{i}\right)
$$

iv) McMullen [22, Theorem 2]:

$$
\mathcal{W}(K) \leq e^{\mathrm{V}_{1}(K)} .
$$

Moreover, in [15] Hadwiger also showed several integral representations of $\mathcal{W}(K)$. We emphasize the following ones (see [15, (1.3) and (1.4)]):

$$
\begin{aligned}
& \mathcal{W}(K)=\int_{\mathbb{R}^{n}} e^{-\pi d(x, K)^{2}} \mathrm{~d} x, \\
& \mathcal{W}(K)=2 \pi \int_{0}^{\infty} \operatorname{vol}\left(K+t B_{2}^{n}\right) t e^{-\pi t^{2}} \mathrm{~d} t .
\end{aligned}
$$

In this paper we obtain new properties of the Wills functional (indeed, we will work in a more general setting that will be stated in Section 2).

The paper is organized as follows. In Section 2 we motivate and introduce, on one hand, an extension of the classical Wills functional in a more general setting, as well as further notation and basic results for functions that will 
be needed later on. On the other hand, we prove some preliminary results as, for instance, the useful property that the Wills functional can be seen as the Asplund product of two log-concave functions (Lemma 2.2), which will be a key result throughout the paper.

Next, Section 3 is devoted to providing upper and lower bounds for the classical Wills functional of a convex body $K$ in terms of other functionals. For instance, $\mathcal{W}(K)$ can be bounded by the volume of $K$ as follows:

Theorem 1.1. Let $K \in \mathcal{K}^{n}$ be a convex body with non-empty interior. Then

$$
\left(8^{n / 2} \operatorname{vol}(K)\right)^{1 / 2} \leq \mathcal{W}(K) \leq \frac{8^{n / 2} \operatorname{vol}(K)}{\sup _{y \in \mathbb{R}^{n}} \gamma_{n}(y+\sqrt{2 \pi} K)}
$$

Here $\gamma_{n}$ denotes the standard Gaussian probability measure on $\mathbb{R}^{n}$, this is,

$$
\mathrm{d} \gamma_{n}(x)=\frac{1}{(2 \pi)^{n / 2}} e^{-\frac{|x|^{2}}{2}} \mathrm{~d} x
$$

We will also reprove McMullen's result (see Theorem A iv)) using a different approach, and will obtain a lower bound for the Wills functional in the same spirit as McMullen's bound. To this end we denote by $\mathrm{R}(K)$ the circumradius of $K$, i.e., $\mathrm{R}(K)=\min \left\{R>0: \exists x \in \mathbb{R}^{n}\right.$ with $\left.K \subset x+R B_{2}^{n}\right\}$.

Theorem 1.2. Let $K \in \mathcal{K}^{n}$. Then

$$
e^{\mathrm{V}_{1}(K)-\pi \mathrm{R}(K)^{2}} \leq \mathcal{W}(K) \leq e^{\mathrm{V}_{1}(K)}
$$

Next, we show that the cube $[-1,1]^{n}$ maximizes the Wills functional among all 0 -symmetric convex bodies in John position. We recall that a convex body $K$ is said to be in John position if the maximum volume ellipsoid contained in $K$ is the Euclidean unit ball.

Theorem 1.3. Let $K \in \mathcal{K}^{n}$ be a 0 -symmetric set in John position. Then

$$
\mathcal{W}(K) \leq \mathcal{W}\left([-1,1]^{n}\right)
$$

In Section 4 we study some Brunn-Minkowski type inequalities for the Wills functional. As we will see, $\mathcal{W}(\cdot)$ is not, in general, $(1 / n)$-concave and so, either less restrictive concavity or additional constants are needed. In this regard we show, among others, the following results:

Theorem 1.4. Let $K, L \in \mathcal{K}^{n}$ and let $\lambda \in(0,1)$. Then

$$
\mathcal{W}((1-\lambda) K+\lambda L) \geq \mathcal{W}(K)^{1-\lambda} \mathcal{W}(L)^{\lambda} .
$$

Theorem 1.5. Let $K, L \in \mathcal{K}^{n}$ and let $\lambda \in(0,1)$. Then

$$
\mathcal{W}((1-\lambda) K+\lambda L)^{1 / n} \geq \frac{1}{(n !)^{1 / n}}\left((1-\lambda) \mathcal{W}(K)^{1 / n}+\lambda \mathcal{W}(L)^{1 / n}\right) .
$$

However, although $\mathcal{W}(\cdot)$ does not satisfy a Brunn-Minkowski inequality in its classical form (i.e., with exponent $1 / n$ ), a reverse Brunn-Minkowski inequality, analogous to the one for the volume proved by Milman [23], holds for the Wills functional: 
Theorem 1.6. Let $K, L \in \mathcal{K}^{n}$. Then there exist $T \in \mathrm{SL}(n)$ and an absolute constant $C>0$ such that

$$
\mathcal{W}(K+T L)^{1 / n} \leq C\left[\mathcal{W}(K)^{1 / n}+\mathcal{W}(L)^{1 / n}\right] .
$$

The last section of the paper is devoted to studying Rogers-Shephard type inequalities for the classical Wills functional. Among others, we get a section/projection Rogers-Shephard type relation for $\mathcal{W}(\cdot)$, as well as an upper bound for the Wills functional of the difference body $K-K:=$ $K+(-K)$.

Theorem 1.7. Let $K \in \mathcal{K}^{n}$ with $0 \in K$ and let $H \in \mathrm{G}(n, k)$. Then

$$
\mathcal{W}\left(P_{H} K\right) \mathcal{W}\left(K \cap H^{\perp}\right) \leq \min \left\{\left(\begin{array}{l}
n \\
k
\end{array}\right) \mathcal{W}(K), 2^{n / 2} \mathcal{W}(\sqrt{2} K)\right\} .
$$

Theorem 1.8. Let $K, L \in \mathcal{K}^{n}$. Then

$$
\mathcal{W}(K \cap L) \mathcal{W}\left(\frac{K-L}{2}\right) \leq 2^{n} \mathcal{W}(K) \mathcal{W}(L) .
$$

In particular we get

$$
\mathcal{W}(K-K) \leq 2^{n} \mathcal{W}(2 K)
$$

2. The generalized Wills functional. Some Preliminary Results

The integral expressions (1.4) showed by Hadwiger in [15] have turned out crucial in many respects. Recently, Kampf [19] proved certain generalizations of them when the 'distance' $d_{E}(x, K)$, between $x \in \mathbb{R}^{n}$ and $K$, relative to a convex body $E$ with $0 \in \operatorname{int} E$, is considered, i.e., for

$$
d_{E}(x, K)=\min _{y \in K}|x-y|_{E}=\min \{t \geq 0: x \in K+t E\}
$$

(see also [18] for the more general case in which the assumption $0 \in \operatorname{int} E$ is not required). He showed that

$$
\int_{\mathbb{R}^{n}} e^{-\pi d_{E}(x, K)^{2}} \mathrm{~d} x=2 \pi \int_{0}^{\infty} \operatorname{vol}(K+t E) t e^{-\pi t^{2}} \mathrm{~d} t=\sum_{i=0}^{n}\left(\begin{array}{c}
n \\
i
\end{array}\right) \frac{\mathrm{W}_{i}(K ; E)}{\kappa_{i}} .
$$

We observe that $f(x)=e^{-\pi d_{E}(x, K)^{2}}$ is a log-concave function because the distance function $d_{E}(\cdot, K)$ is convex (cf. e.g. [25, Lemma 1.5.9]); we recall that $f: \mathbb{R}^{n} \longrightarrow \mathbb{R}_{\geq 0}$ is said to be log-concave if

$$
f((1-\lambda) x+\lambda y) \geq f(x)^{1-\lambda} f(y)^{\lambda} \quad \text { for any } \lambda \in(0,1) \text { and all } x, y \in \mathbb{R}^{n},
$$

or equivalently, if it is of the form

$$
f(x)=e^{-u(x)}
$$

for $u: \mathbb{R}^{n} \longrightarrow \mathbb{R} \cup\{\infty\}$ a convex function.

The study of log-concave functions has become very important in the recent years, among others in the study of problems related to the distribution of mass in a convex body (we refer the reader, for instance, to $[13,20]$ and 
the references therein). Notice also that convex bodies are contained inside the class of log-concave functions via either the negative exponential of the Minkowski functional $|\cdot|_{K}$ of a convex body $K$ (whose integral is, up to a constant, the volume), or its characteristic function, which we will denote throughout the paper by $\chi_{K}$, i.e.,

$$
\chi_{K}(x)= \begin{cases}1 & \text { if } x \in K \\ 0 & \text { otherwise. }\end{cases}
$$

Going back to the integral representation (2.2), we observe that a more general functional can be obtained by replacing $e^{-\pi t^{2}}$ by another function $G(t)$ properly associated to a log-concave measure $\mu$ on the non-negative real line $\mathbb{R}_{\geq 0}$. Thus, let $\mu$ be the measure on $\mathbb{R}_{\geq 0}$ given by $\mathrm{d} \mu(t)=\phi(t) \mathrm{d} t$, where $\phi: \mathbb{R}_{\geq 0} \longrightarrow \mathbb{R}_{\geq 0}$ is log-concave. Then, the associated function $G(t)=$ $\mu([t, \infty))$ is decreasing and log-concave, and hence $G(t)=e^{-u(t)}$ for a convex function $u: \mathbb{R}_{>0} \longrightarrow \mathbb{R} \cup\{\infty\}$ which is increasing and, without loss of generality, can be assumed to be continuous in $\left\{t \in \mathbb{R}_{\geq 0}: u(t) \neq \infty\right\}$. Moreover, if $\mu$ is a probability measure, then $G(0)=1$, i.e., $u(0)=0$.

Thus, we may consider the more general expression

$$
f_{K, E}^{u}(x)=G\left(d_{E}(x, K)\right)=e^{-u\left(d_{E}(x, K)\right)} \quad \text { for } K, E \in \mathcal{K}^{n},
$$

where $u: \mathbb{R}_{\geq 0} \longrightarrow \mathbb{R} \cup\{\infty\}$ satisfies the above assumptions (monotonicity, continuity and convexity). From now on, and for the sake of brevity, we will denote by $\mathfrak{C}\left(\mathbb{R}_{\geq 0}\right)$ the family of all convex functions $u: \mathbb{R}_{\geq 0} \longrightarrow \mathbb{R} \cup\{\infty\}$ which are continuous in $\left\{t \in \mathbb{R}_{\geq 0}: u(t) \neq \infty\right\}$ and increasing.

The desired outcome that integration of our function $f_{K, E}^{u}$ provides a Steiner type formula (with weights) indeed holds (see also [19, Proposition 3] and [18, Lemma 1.1]).

Proposition 2.1. Let $K, E \in \mathcal{K}^{n}$ with $0 \in \operatorname{int} E$ and let $u \in \mathfrak{C}\left(\mathbb{R}_{\geq 0}\right)$ be strictly increasing. Then

$$
\int_{\mathbb{R}^{n}} f_{K, E}^{u}(x) \mathrm{d} x=\int_{u(0)}^{\infty} \operatorname{vol}\left(K+u^{-1}(s) E\right) e^{-s} \mathrm{~d} s=\sum_{i=0}^{n}\left(\begin{array}{c}
n \\
i
\end{array}\right) \mathfrak{m}_{i}^{u} \mathrm{~W}_{i}(K ; E),
$$

where

$$
\mathfrak{m}_{i}^{u}=\int_{u(0)}^{\infty} u^{-1}(s)^{i} e^{-s} \mathrm{~d} s
$$

We notice that when dealing with a differentiable function $u$, the numbers $\mathfrak{m}_{i}^{u}$ are nothing else but the moments of the measure $\mu$ associated to $u$, i.e., such that $G(t)=\mu([t, \infty))=e^{-u(t)}$ :

$$
\mathfrak{m}_{i}^{u}=\int_{u(0)}^{\infty} u^{-1}(s)^{i} e^{-s} \mathrm{~d} s=\int_{0}^{\infty} t^{i} \mathrm{~d} \mu(t) .
$$


FURTHER INEQUALITIES FOR THE (GENERALIZED) WILLS FUNCTIONAL 7

Proof of Proposition 2.1. Using Fubini's theorem we get

$$
\begin{aligned}
\int_{\mathbb{R}^{n}} f_{K, E}^{u}(x) \mathrm{d} x & =\int_{\mathbb{R}^{n}} e^{-u\left(d_{E}(x, K)\right)} \mathrm{d} x=\int_{\mathbb{R}^{n}} \int_{u\left(d_{E}(x, K)\right)}^{\infty} e^{-s} \mathrm{~d} s \mathrm{~d} x \\
& =\int_{u(0)}^{\infty} e^{-s} \int_{\mathbb{R}^{n}} \chi_{\left\{y \in \mathbb{R}^{n}: u\left(d_{E}(y, K)\right) \leq s\right\}}(x) \mathrm{d} x \mathrm{~d} s .
\end{aligned}
$$

Now, the strict monotonicity of $u(t)$ ensures the existence of the inverse $u^{-1}(s)$, and hence, by (2.1),

$$
\begin{aligned}
\left\{x \in \mathbb{R}^{n}: u\left(d_{E}(x, K)\right) \leq s\right\} & =\left\{x \in \mathbb{R}^{n}: d_{E}(x, K) \leq u^{-1}(s)\right\} \\
& =K+u^{-1}(s) E .
\end{aligned}
$$

Thus, via the Steiner formula (1.1) we can conclude that

$$
\begin{aligned}
\int_{\mathbb{R}^{n}} f_{K, E}^{u}(x) \mathrm{d} x & =\int_{u(0)}^{\infty} e^{-s} \int_{\mathbb{R}^{n}} \chi_{\left\{y \in \mathbb{R}^{n}: u\left(d_{E}(y, K)\right) \leq s\right\}}(x) \mathrm{d} x \mathrm{~d} s \\
& =\int_{u(0)}^{\infty} \operatorname{vol}\left(K+u^{-1}(s) E\right) e^{-s} \mathrm{~d} s \\
& =\int_{u(0)}^{\infty} \sum_{i=0}^{n}\left(\begin{array}{c}
n \\
i
\end{array}\right) \mathrm{W}_{i}(K ; E) u^{-1}(s)^{i} e^{-s} \mathrm{~d} s \\
& =\sum_{i=0}^{n}\left[\left(\begin{array}{c}
n \\
i
\end{array}\right) \mathrm{W}_{i}(K ; E) \int_{u(0)}^{\infty} u^{-1}(s)^{i} e^{-s} \mathrm{~d} s\right] .
\end{aligned}
$$

Example 2.1. (i) The classical Wills functional $\mathcal{W}(K)$ is obtained for $f_{K, B_{2}^{n}}^{\pi(\cdot)^{2}}$ (cf. (1.3)) because, clearly, the moments

$$
\mathfrak{m}_{i}^{\pi(\cdot)^{2}}=\frac{1}{\kappa_{i}}
$$

(ii) In the case of the function $u_{p}(t)=(2 \Gamma(1+1 / p) t)^{p}, p>1$, we have

$$
\int_{u_{p}(0)}^{\infty} u_{p}^{-1}(s)^{i} e^{-s} \mathrm{~d} s=\int_{0}^{\infty} \frac{s^{i / p}}{2^{i} \Gamma\left(1+\frac{1}{p}\right)^{i}} e^{-s} \mathrm{~d} s=\frac{\Gamma\left(1+\frac{i}{p}\right)}{\left(2 \Gamma\left(1+\frac{1}{p}\right)\right)^{i}}=\frac{1}{\operatorname{vol}_{i}\left(B_{p}^{i}\right)},
$$

where $B_{p}^{i}$ is the unit $p$-ball associated to the $p$-norm $|x|_{p}=\left(\sum_{i=1}^{n}\left|x_{i}\right|^{p}\right)^{1 / p}$. So, we get

$$
\int_{\mathbb{R}^{n}} f_{K, E}^{u_{p}}(x) \mathrm{d} x=\sum_{i=0}^{n}\left(\begin{array}{c}
n \\
i
\end{array}\right) \frac{1}{\operatorname{vol}_{i}\left(B_{p}^{i}\right)} \mathrm{W}_{i}(K ; E) .
$$

As mentioned in the introduction, $\mathcal{W}(K)$ depends only on the convex body, but not on the dimension of the embedding space. However, in the most general case of the function $f_{K, E}^{u}$, it does not come true anymore. Therefore, we need a special notation which allows us to distinguish the dimension in which the Wills functional is computed, as well as the involved 
sets. Thus, if $K, E \in \mathcal{K}^{n}$ with $\operatorname{dim} K=\operatorname{dim} E=k$ and we compute the extended Wills functional in $\mathbb{R}^{k}$ (assuming that both $K, E$ lie in the same affine $k$-dimensional space), we will write

$$
\mathcal{W}_{u}^{(k)}(K ; E):=\int_{\mathbb{R}^{k}} f_{K, E}^{u}(x) \mathrm{d} x,
$$

whereas the $n$-dimensional functional will be denoted just by $\mathcal{W}_{u}(K ; E)$. Moreover, we will keep the classical notation for the usual Wills functional, i.e., $\mathcal{W}(K):=\mathcal{W}_{\pi(\cdot)^{2}}\left(K ; B_{2}^{n}\right)$

2.1. Some basics on (log-concave) functions. Since log-concave functions are the keystone of the coming development, we need to recall some background.

A first major result for (measurable) functions is the so-called BorellBrascamp-Lieb inequality (see e.g. [13, Theorem 10.1]):

Theorem B (Borell-Brascamp-Lieb's inequality). Let $\lambda \in(0,1)$ be fixed, let $-1 / n \leq p \leq \infty$ and let $f, g, h: \mathbb{R}^{n} \longrightarrow \mathbb{R}_{\geq 0}$ be non-negative measurable functions such that, for any $x, y \in \mathbb{R}^{n}$ with $f \overline{(x)} g(y)>0$,

$$
h((1-\lambda) x+\lambda y) \geq\left((1-\lambda) f(x)^{p}+\lambda g(y)^{p}\right)^{1 / p} .
$$

Then

$$
\int_{\mathbb{R}^{n}} h(x) \mathrm{d} x \geq\left[(1-\lambda)\left(\int_{\mathbb{R}^{n}} f(x) \mathrm{d} x\right)^{q}+\lambda\left(\int_{\mathbb{R}^{n}} g(x) \mathrm{d} x\right)^{q}\right]^{1 / q},
$$

provided that $f$ and $g$ have non-zero integrals, and where $q=p /(n p+1)$.

The case $p=0$ of the Borell-Brascamp-Lieb inequality is known as the Prékopa-Leindler inequality: if $h((1-\lambda) x+\lambda y) \geq f(x)^{1-\lambda} g(y)^{\lambda}$ then

$$
\int_{\mathbb{R}^{n}} h(x) \mathrm{d} x \geq\left(\int_{\mathbb{R}^{n}} f(x) \mathrm{d} x\right)^{1-\lambda}\left(\int_{\mathbb{R}^{n}} g(x) \mathrm{d} x\right)^{\lambda} .
$$

For $p \geq 1$, we recall that the $p$-norm of a function $f: \mathbb{R}^{n} \longrightarrow \mathbb{R}_{\geq 0}$ is defined as

$$
\|f\|_{p}=\left(\int_{\mathbb{R}^{n}} f(x)^{p} \mathrm{~d} x\right)^{1 / p}
$$

where the case $p=\infty$ has to be understood as

$$
\|f\|_{\infty}=\sup _{x \in \mathbb{R}^{n}} f(x) .
$$

Next we set the definition of three important operations for functions: convolution, Asplund product and difference function.

Definition 2.1. The Asplund product of two functions $f, g: \mathbb{R}^{n} \longrightarrow \mathbb{R}_{\geq 0}$ is given by

$$
(f \star g)(z)=\sup _{z=x+y} f(x) g(y)
$$


and their convolution is defined as

$$
(f * g)(z)=\int_{\mathbb{R}^{n}} f(x) g(z-x) \mathrm{d} x .
$$

For $\lambda \in(0,1)$, the $\lambda$-difference function associated with $f$ and $g$ is given by

$$
\Delta_{\lambda}^{f, g}(z)=\sup _{z=(1-\lambda) x+\lambda y} f\left(\frac{x}{1-\lambda}\right)^{1-\lambda} g\left(\frac{-y}{\lambda}\right)^{\lambda} .
$$

Notice that in the case of characteristic functions of two convex bodies $K, L \in \mathcal{K}^{n}$, the convolution $\chi_{K} * \chi_{L}=\operatorname{vol}(K \cap(\cdot-L))$ whereas the Asplund product $\chi_{K} \star \chi_{E}=\chi_{K+E}$, and so it plays the role of the Minkowski addition of convex bodies in the setting of log-concave functions. In the last decades, many efforts have been made in order to extend geometric inequalities for convex bodies to the more general setting of log-concave functions.

In $\left[5\right.$, Theorem 1.8] it is proved that if $f, g: \mathbb{R}^{n} \longrightarrow \mathbb{R}_{>0}$ are log-concave functions and $\lambda \in(0,1)$, then the following Rogers-Shephard type inequality holds:

$$
\int_{\mathbb{R}^{n}} f(x)^{\lambda} g(x)^{1-\lambda} \mathrm{d} x \int_{\mathbb{R}^{n}} \Delta_{\lambda}^{f, g}(z) \mathrm{d} z \leq \int_{\mathbb{R}^{n}} f(x) \mathrm{d} x \int_{\mathbb{R}^{n}} g(y) \mathrm{d} y .
$$

Moreover (see [4, Theorem 2.1]),

$$
\|f * g\|_{\infty} \int_{\mathbb{R}^{n}}(f \star g)(x) \mathrm{d} x \leq\left(\begin{array}{c}
2 n \\
n
\end{array}\right)\|f\|_{\infty}\|g\|_{\infty} \int_{\mathbb{R}^{n}} f(x) \mathrm{d} x \int_{\mathbb{R}^{n}} g(x) \mathrm{d} x .
$$

Another outstanding result for log-concave functions, providing a reverse Brunn-Minkowski inequality, was obtained by Klartag and Milman in [20, Theorem 1.3 and Remark (2) in page 181]:

Theorem C. Let $f, g: \mathbb{R}^{n} \longrightarrow \mathbb{R}_{\geq 0}$ be log-concave functions with finite and positive integrals, such that $\|f\|_{\infty}=\|g\|_{\infty}=f(0)=g(0)=1$. Then there exist $T_{1}, T_{2} \in \mathrm{SL}(n)$ and an absolute constant $C>0$ such that

$$
\begin{aligned}
&\left(\int _ { \mathbb { R } ^ { n } } \left(\left(f \circ T_{1}\right)\right.\right.\left.\left.\star\left(g \circ T_{2}\right)\right)(x) \mathrm{d} x\right)^{1 / n} \\
& \leq C\left[\left(\int_{\mathbb{R}^{n}}\left(f \circ T_{1}\right)(x) \mathrm{d} x\right)^{1 / n}+\left(\int_{\mathbb{R}^{n}}\left(g \circ T_{2}\right)(x) \mathrm{d} x\right)^{1 / n}\right] .
\end{aligned}
$$

We conclude this subsection recalling one last concept: the projection of a function (see e.g. [20]).

Definition 2.2. Let $f: \mathbb{R}^{n} \longrightarrow \mathbb{R}_{\geq 0}$ and let $H \in \mathrm{G}(n, k)$. The projection of $f$ onto $H$ is the function $P_{H} f: H \longrightarrow \mathbb{R}_{\geq 0} \cup\{\infty\}$ defined by

$$
\left(P_{H} f\right)(x)=\sup _{y \in H^{\perp}} f(x+y) .
$$

The geometric meaning of this definition is easy: the (strict) hypograph of $P_{H} f$ is the projection of the (strict) hypograph of $f$ onto $H$. In particular, for $K \in \mathcal{K}^{n}, P_{H} \chi_{K}=\chi_{P_{H} K}$. 
Regarding the projection of a function $f: \mathbb{R}^{n} \longrightarrow \mathbb{R}_{\geq 0}$, very recently $[1$, Theorems 1.1 and 1.2] the following Rogers-Shephard type inequalities for log-concave (integrable) functions have been obtained: for $H \in \mathrm{G}(n, k)$,

$$
\int_{H}\left(P_{H} f\right)(x) \mathrm{d} x \int_{H^{\perp}} f(y) \mathrm{d} y \leq\left(\begin{array}{l}
n \\
k
\end{array}\right)\|f\|_{\infty} \int_{\mathbb{R}^{n}} f(z) \mathrm{d} z ;
$$

moreover, if $\|f\|_{\infty}=f(0)$ then, for any $\lambda \in(0,1)$,

$$
(1-\lambda)^{k} \lambda^{n-k} \int_{H}\left(P_{H} f\right)(x)^{1-\lambda} \mathrm{d} x \int_{H^{\perp}} f(y)^{\lambda} \mathrm{d} y \leq \int_{\mathbb{R}^{n}} f(z) \mathrm{d} z .
$$

2.2. Some preliminary lemmas. We start this subsection showing several properties for the Asplund product that will be needed later on.

Lemma 2.1. Let $f, g: \mathbb{R}^{n} \longrightarrow \mathbb{R}_{>0}$ be two non-negative functions, and let $T_{1}, T_{2} \in \mathrm{SL}(n)$. Then, for all $x \in \overline{\mathbb{R}}^{n}$,

$$
\left(\left(f \circ T_{1}\right) \star\left(g \circ T_{2}\right)\right)(x)=\left(f \star\left(g \circ\left(T_{2} T_{1}^{-1}\right)\right)\right)\left(T_{1}(x)\right) .
$$

Proof. With the changes of variable $w=T_{1} y$ and $z=T_{1} x$, we get

$$
\begin{aligned}
\left(\left(f \circ T_{1}\right) \star\left(g \circ T_{2}\right)\right)(x) & =\sup _{y \in \mathbb{R}^{n}} f\left(T_{1} y\right) g\left(T_{2}(x-y)\right) \\
& =\sup _{w \in \mathbb{R}^{n}} f(w) g\left(T_{2} x-T_{2} T_{1}^{-1} w\right) \\
& =\sup _{w \in \mathbb{R}^{n}} f(w) g\left(T_{2} T_{1}^{-1}(z-w)\right) \\
& =\left(f \star\left(g \circ\left(T_{2} T_{1}^{-1}\right)\right)\right)\left(T_{1}(x)\right) .
\end{aligned}
$$

The function $f_{K, E}^{u}$ defining the generalized Wills functional can be seen as the Asplund product of two log-concave functions. This property will be helpful in the subsequent results.

Lemma 2.2. Let $u \in \mathfrak{C}\left(\mathbb{R}_{\geq 0}\right)$ and let $K, E \in \mathcal{K}^{n}$ with $0 \in \operatorname{int} E$. Then

$$
f_{K, E}^{u}=e^{-u\left(|\cdot|_{E}\right)} \star \chi_{K} .
$$

Proof. By definition of Asplund product and since $u$ is increasing,

$$
\begin{aligned}
\left(e^{-u\left(|\cdot|_{E}\right)} \star \chi_{K}\right)(x) & =\sup _{y \in \mathbb{R}^{n}} e^{-u\left(|x-y|_{E}\right)} \chi_{K}(y) \\
& =\sup _{y \in K} e^{-u\left(|x-y|_{E}\right)}=e^{-\inf _{y \in K} u\left(|x-y|_{E}\right)} \\
& =e^{-u\left(\inf _{y \in K}|x-y|_{E}\right)}=e^{-u\left(d_{E}(x, K)\right)}
\end{aligned}
$$

for all $x \in \mathbb{R}^{n}$.

Also the projection of a function "behaves well" with our function $f_{K, E}^{u}$ : 
FURTHER INEQUALITIES FOR THE (GENERALIZED) WILLS FUNCTIONAL 11

Lemma 2.3. Let $K, E \in \mathcal{K}^{n}$ with $0 \in \operatorname{int} E, u \in \mathfrak{C}\left(\mathbb{R}_{\geq 0}\right)$ and $H \in \mathrm{G}(n, k)$. Then, for every $x \in H$,

$$
\left(P_{H} f_{K, E}^{u}\right)(x)=f_{P_{H} K, P_{H} E}^{u}(x) .
$$

Moreover, $f_{K, E}^{u}(x) \geq f_{K \cap H, E \cap H}^{u}(x)$.

Proof. If we show that

$$
d_{P_{H} E}\left(x, P_{H} K\right)=\inf _{y \in H^{\perp}} d_{E}(x+y, K),
$$

then we immediately get the first equality, because in such case

$$
\begin{aligned}
\left(P_{H} f_{K, E}^{u}\right)(x) & =\sup _{y \in H^{\perp}} f_{K, E}^{u}(x+y)=\sup _{y \in H^{\perp}} e^{-u\left(d_{E}(x+y, K)\right)} \\
& =e^{-\inf _{y \in H^{\perp}} u\left(d_{E}(x+y, K)\right)}=e^{-u\left(\inf _{y \in H^{\perp}} d_{E}(x+y, K)\right)} \\
& =e^{-u\left(d_{P_{H} E}\left(x, P_{H} K\right)\right)}=f_{P_{H} K, P_{H} E}^{u}(x) .
\end{aligned}
$$

So, we have to prove (2.11). Let $x \in H$ and $y \in H^{\perp}$. If $x+y \in K+t E$ then $x=P_{H}(x+y) \in P_{H}(K+t E)=P_{H} K+t P_{H} E$, and hence

$$
\{t \geq 0: x+y \in K+t E\} \subset\left\{t \geq 0: x \in P_{H} K+t P_{H} E\right\} .
$$

Therefore, from the definition of $d_{E}$ (see (2.1)) we conclude that

$$
d_{E}(x+y, K) \geq d_{P_{H} E}\left(x, P_{H} K\right) \quad \text { for all } y \in H^{\perp} .
$$

Now, let $t_{0}=d_{P_{H} E}\left(x, P_{H} K\right)$. Then $x \in P_{H} K+t_{0} P_{H} E=P_{H}\left(K+t_{0} E\right)$ and so there exists $y_{0} \in H^{\perp}$ such that $x+y_{0} \in K+t_{0} E$. This implies that $t_{0} \geq d_{E}\left(x+y_{0}, K\right)$ which, together with (2.12), yields $t_{0}=d_{E}\left(x+y_{0}, K\right)$. Then we can conclude that

$$
\inf _{y \in H^{\perp}} d_{E}(x+y, K)=t_{0}=d_{P_{H} E}\left(x, P_{H} K\right),
$$

as required.

The proof of the second assertion of the lemma is straightforward: since $(K \cap H)+t(E \cap H) \subset K+t E,(2.1)$ yields $d_{E \cap H}(x, K \cap H) \geq d_{E}(x, K)$, and so

$$
f_{K, E}^{u}(x)=e^{-u\left(d_{E}(x, K)\right)} \geq e^{-u\left(d_{E \cap H}(x, K \cap H)\right)}=f_{K \cap H, E \cap H}^{u}(x) .
$$

Finally, we compute the $p$-norm of the function $f_{K, E}^{u}$. Given $K, E \in \mathcal{K}^{n}$ with $0 \in \operatorname{int} E, u \in \mathfrak{C}\left(\mathbb{R}_{\geq 0}\right)$ and $1 \leq p<\infty$, we clearly have

$$
\left\|f_{K, E}^{u}\right\|_{p}=\left(\int_{\mathbb{R}^{n}} e^{-p u\left(d_{E}(x, K)\right)} \mathrm{d} x\right)^{1 / p}=\mathcal{W}_{p u}(K ; E)^{1 / p} .
$$

In particular, for $u=\pi(\cdot)^{2}$ and $E=B_{2}^{n}$, we get the following relation.

Lemma 2.4. Let $K \in \mathcal{K}^{n}$ and $1 \leq p<\infty$. Then

$$
\left\|f_{K, B_{2}^{n}}^{\pi(\cdot)^{2}}\right\|_{p}=\frac{1}{p^{n /(2 p)}} \mathcal{W}(\sqrt{p} K)^{1 / p}
$$


Proof. Doing the change of variable $\sqrt{p} x=y$, we immediately get

$$
\begin{aligned}
\left\|f_{K, B_{2}^{n}}^{\pi(\cdot)^{2}}\right\|_{p} & =\left(\int_{\mathbb{R}^{n}} e^{-p \pi d(x, K)^{2}} \mathrm{~d} x\right)^{1 / p}=\left(\int_{\mathbb{R}^{n}} e^{-\pi d(\sqrt{p} x, \sqrt{p} K)^{2}} \mathrm{~d} x\right)^{1 / p} \\
& =\left(\frac{1}{p^{n / 2}} \int_{\mathbb{R}^{n}} e^{-\pi d(y, \sqrt{p} K)^{2}} \mathrm{~d} y\right)^{1 / p}=\frac{1}{p^{n /(2 p)}} \mathcal{W}(\sqrt{p} K)^{1 / p} .
\end{aligned}
$$

\section{Bounding the Wills functional of a COnVEX Body}

In this section we provide upper and lower bounds for the Wills functional of a convex body $K$ in terms of other functionals. Our first aim will be to relate $\mathcal{W}_{u}(K ; E)$ with the volumes of the involved sets.

3.1. Relating the Wills functional to the volume. We start this subsection by showing Theorem 1.1, which will be a consequence of a more general result for $\mathcal{W}_{u}(K ; E)$. First we study the lower bound.

Theorem 3.1. Let $K, E \in \mathcal{K}^{n}$ with $0 \in \operatorname{int} E$ and let $u \in \mathfrak{C}\left(\mathbb{R}_{\geq 0}\right)$. Then, for any $\lambda \in(0,1)$,

$$
\mathcal{W}_{u}(K ; E) \geq\left(\frac{1}{\lambda^{\lambda}(1-\lambda)^{1-\lambda}}\right)^{n}\left(\int_{\mathbb{R}^{n}} e^{-\frac{u\left(|x|_{E}\right)}{1-\lambda}} \mathrm{d} x\right)^{1-\lambda} \operatorname{vol}(K)^{\lambda} .
$$

In particular, for $u=\pi(\cdot)^{2}$ and $E=B_{2}^{n}$, we have

$$
\mathcal{W}(K) \geq\left(\frac{1}{\lambda^{\lambda}(1-\lambda)^{(1-\lambda) / 2}}\right)^{n} \operatorname{vol}(K)^{\lambda} .
$$

Proof. We consider the functions

$$
f=e^{-u\left((1-\lambda)|\cdot|_{E}\right) /(1-\lambda)}, \quad g=\chi_{K / \lambda} \quad \text { and } \quad h=f\left(\frac{\cdot}{1-\lambda}\right)^{1-\lambda} \star g\left(\frac{\cdot}{\lambda}\right)^{\lambda},
$$

for which $h=e^{-u\left(|\cdot|_{E}\right)} \star \chi_{K}=f_{K, E}^{u}$ by Lemma 2.2. Then, the PrékopaLeindler inequality (Theorem B for $p=0$ ) applied to $f, g$ and $h$ yields (3.1).

When $E=B_{2}^{n}$ and $u=\pi(\cdot)^{2}$, and since the standard Gaussian measure $\gamma_{n}$ is a probability measure, we get (cf. (1.5))

$$
\int_{\mathbb{R}^{n}} e^{-\frac{\pi}{1-\lambda}|x|^{2}} \mathrm{~d} x=\frac{(1-\lambda)^{n / 2}}{(2 \pi)^{n / 2}} \int_{\mathbb{R}^{n}} e^{-\frac{|x|^{2}}{2}} \mathrm{~d} x=(1-\lambda)^{n / 2}
$$

This finishes the proof.

Following the idea of the above proof but now using the linear refinement of the Prékopa-Leindler inequality (see [12, Theorem 1.5]), we immediately get the following improvement of (3.2) for the classical Wills functional.

Corollary 3.1. Let $K \in \mathcal{K}^{n}$ be such that there exists $H \in \mathrm{G}(n, n-1)$ with

$$
\operatorname{vol}_{n-1}\left(P_{H} K\right)=\left(\frac{\lambda}{\sqrt{1-\lambda}}\right)^{n-1}
$$


for some $\lambda \in(0,1)$. Then

$$
\mathcal{W}(K) \geq \frac{\operatorname{vol}(K)}{\lambda^{n-1}}+\frac{1}{(1-\lambda)^{(n-2) / 2}} .
$$

Proof. Given two log-concave functions $f, g: \mathbb{R}^{n} \longrightarrow \mathbb{R}_{\geq 0}$ (decaying to zero at infinity), if there exists a hyperplane $H \in \mathrm{G}(n, n-1)$ such that $P_{H} f$ and $P_{H} g$ have the same (finite) integral, then the right-hand side in the Prékopa-Leindler inequality (2.6) (Theorem B for $p=0$ ) can be replaced by the arithmetic mean of the integrals of $f$ and $g$ (see [12, Theorem 1.5]):

$$
\int_{\mathbb{R}^{n}} h(x) \mathrm{d} x \geq(1-\lambda) \int_{\mathbb{R}^{n}} f(x) \mathrm{d} x+\lambda \int_{\mathbb{R}^{n}} g(x) \mathrm{d} x .
$$
Since

Let $L=(\sqrt{1-\lambda} / \lambda) K, f=e^{-\pi|\cdot|^{2}}, g=\chi_{L}$ and $h=f\left(\frac{\cdot}{1-\lambda}\right)^{1-\lambda} \star g(\dot{\bar{\lambda}})^{\lambda}$.

$$
\left(P_{H} f\right)(x)=\sup _{y \in H^{\perp}} e^{-\pi|x+y|^{2}}=e^{-\pi|x|^{2}} \sup _{y \in H^{\perp}} e^{-\pi|y|^{2}}=e^{-\pi|x|^{2}}
$$

for all $x \in H$, then (cf.(3.3))

$$
\int_{H}\left(P_{H} f\right)(x) \mathrm{d} x=1=\operatorname{vol}_{n-1}\left(P_{H} L\right)=\int_{H}\left(P_{H} g\right)(x) \mathrm{d} x,
$$

and hence we are in the above conditions and we can apply the mentioned refinement of the Prékopa-Leindler inequality. Thus, using Lemma 2.2,

$$
h=e^{-\frac{\pi}{1-\lambda}|\cdot|^{2}} \star \chi_{\lambda L}=e^{-\frac{\pi}{1-\lambda} d(\cdot, \lambda L)^{2}}=e^{-\pi d\left(\frac{\cdot}{\sqrt{1-\lambda}}, K\right)^{2}},
$$

and so, integrating $f, g$ and $h$, doing the change of variable $y=x / \sqrt{1-\lambda}$ and applying (3.4), we get

$$
(1-\lambda)^{n / 2} \mathcal{W}(K) \geq 1-\lambda+\lambda \operatorname{vol}(L)=1-\lambda+\frac{(1-\lambda)^{n / 2}}{\lambda^{n-1}} \operatorname{vol}(K) .
$$

This finishes the proof.

By taking $\lambda=1 / 2$ in (3.2), we obtain the lower bound in Theorem 1.1:

$$
\mathcal{W}(K) \geq\left(8^{n / 2} \operatorname{vol}(K)\right)^{1 / 2} \text {. }
$$

Next we show the upper bound in Theorem 1.1: up to a factor depending on the standard Gaussian measure $\gamma_{n}$ of $K$, the (classical) Wills functional $\mathcal{W}(\cdot)$ can be bounded from above by $8^{n / 2} \operatorname{vol}(K)$. This is the content of the following result in the more general setting of the generalized Wills functional $\mathcal{W}_{u}(\cdot ; E)$.

Theorem 3.2. Let $K, E \in \mathcal{K}^{n}$ be convex bodies with non-empty interior such that $0 \in \operatorname{int} E$ and let $u \in \mathfrak{C}\left(\mathbb{R}_{\geq 0}\right)$ be strictly increasing. Then

$$
\mathcal{W}_{u}(K ; E) \leq \min \left\{\left(\begin{array}{c}
2 n \\
n
\end{array}\right) e^{-u(0)} \mathfrak{m}_{n}^{u}, 4^{n} \mathfrak{m}_{n}^{2 u}\right\} \frac{\operatorname{vol}(K) \operatorname{vol}(E)}{\sup _{y \in \mathbb{R}^{n}} \mu_{u, E}(y-K)},
$$


where $\mu_{u, E}$ is the measure on $\mathbb{R}^{n}$ given by $\mathrm{d} \mu_{u, E}(x)=e^{-u\left(|x|_{E}\right)} \mathrm{d} x$. In particular, for $u=\pi(\cdot)^{2}$ and $E=B_{2}^{n}$, we have

$$
\mathcal{W}(K) \leq \frac{\min \left\{\left(\begin{array}{c}
2 n \\
n
\end{array}\right), 8^{n / 2}\right\} \operatorname{vol}(K)}{\sup _{y \in \mathbb{R}^{n}} \gamma_{n}(y+\sqrt{2 \pi} K)},
$$

where $C_{n}:=\min \left\{\left(\begin{array}{c}2 n \\ n\end{array}\right), 8^{n / 2}\right\}$ is given by $C_{2}=6, C_{3}=20$ and $C_{n}=8^{n / 2}$ for all $n \geq 4$.

Proof. Let $f=e^{-u\left(|\cdot|_{E}\right)}$ and let $g=\chi_{K}$. Then, by Lemma 2.2, we have that $f \star g=f_{K, E}^{u}$. Moreover, $\|f\|_{\infty}=e^{-u(0)}$,

$$
(f * g)(y)=\int_{\mathbb{R}^{n}} e^{-u\left(|x|_{E}\right)} \chi_{K}(y-x) \mathrm{d} x=\int_{y-K} e^{-u\left(|x|_{E}\right)} \mathrm{d} x
$$

and

$$
\begin{aligned}
\int_{\mathbb{R}^{n}} f(x) \mathrm{d} x & =\int_{\mathbb{R}^{n}} e^{-u\left(|x|_{E}\right)} \mathrm{d} x=\int_{\mathbb{R}^{n}} \int_{u\left(|x|_{E}\right)}^{\infty} e^{-s} \mathrm{~d} s \mathrm{~d} x \\
& =\int_{u(0)}^{\infty} e^{-s} \int_{\mathbb{R}^{n}} \chi_{\left\{y: u\left(|y|_{E}\right) \leq s\right\}}(x) \mathrm{d} x \mathrm{~d} s \\
& =\int_{u(0)}^{\infty} e^{-s} \operatorname{vol}\left(u^{-1}(s) E\right) \mathrm{d} s=\mathfrak{m}_{n}^{u} \operatorname{vol}(E) .
\end{aligned}
$$

On one hand, by applying (2.8) to the functions $f$ and $g$ we get

$$
\sup _{y \in \mathbb{R}^{n}} \mu_{u, E}(y-K) \mathcal{W}_{u}(K ; E) \leq\left(\begin{array}{c}
2 n \\
n
\end{array}\right) e^{-u(0)} \mathfrak{m}_{n}^{u} \operatorname{vol}(K) \operatorname{vol}(E) .
$$

On the other hand, by applying (2.7) for $\lambda=1 / 2$ to the functions $f$ and $g$, we obtain

$$
\begin{aligned}
\mu_{u / 2, E}(-K) \mathcal{W}_{u / 2}(K ; E) & =\int_{-K} e^{-\frac{u}{2}\left(|x|_{E}\right)} \mathrm{d} x \int_{\mathbb{R}^{n}} f_{K, E}^{u / 2}(x) \mathrm{d} x \\
& =\int_{\mathbb{R}^{n}} \sqrt{f(x) g(-x)} \mathrm{d} x \int_{\mathbb{R}^{n}} \sqrt{f \star g(x)} \mathrm{d} x \\
& \leq 4^{n} \mathfrak{m}_{n}^{u} \operatorname{vol}(K) \operatorname{vol}(E) .
\end{aligned}
$$

Since both $\mathcal{W}_{u / 2}(\cdot ; E)$ and $\operatorname{vol}(\cdot)$ are translation invariant we get (from the above inequality for $2 u$ ) that

$$
\sup _{y \in \mathbb{R}^{n}} \mu_{u, E}(y-K) \mathcal{W}_{u}(K ; E) \leq 4^{n} \mathfrak{m}_{n}^{2 u} \operatorname{vol}(K) \operatorname{vol}(E),
$$

which, together with (3.8), shows (3.5).

When $E=B_{2}^{n}$ and $u=\pi(\cdot)^{2}$, since

$$
\mathfrak{m}_{n}^{2 \pi(\cdot)^{2}}=\frac{1}{(2 \pi)^{n / 2}} \int_{0}^{\infty} e^{-s} s^{n / 2} \mathrm{~d} s=\frac{\mathfrak{m}_{n}^{\pi(\cdot)^{2}}}{2^{n / 2}}=\frac{1}{2^{n / 2}} \frac{1}{\kappa_{n}}
$$


(see (2.5)) and $\mu_{\pi(\cdot)^{2}, B_{2}^{n}}(y-K)=\gamma_{n}(-\sqrt{2 \pi} y+\sqrt{2 \pi} K)$, then (3.5) yields (3.6). Finally, since $\left(\begin{array}{c}2 n \\ n\end{array}\right) \geq\left(\begin{array}{c}2 n \\ i\end{array}\right)$ for all $i=0, \ldots, 2 n$, we have that

$$
\left(\begin{array}{c}
2 n \\
n
\end{array}\right) \geq \frac{1}{2 n+1} \sum_{i=0}^{2 n}\left(\begin{array}{c}
2 n \\
i
\end{array}\right)=\frac{4^{n}}{2 n+1},
$$

which, jointly with the fact that $2^{n / 2} \geq 2 n+1$ for all $n \geq 9$, implies that

$$
C_{n}=\min \left\{\left(\begin{array}{c}
2 n \\
n
\end{array}\right), 8^{n / 2}\right\}=8^{n / 2} \quad \text { for all } n \geq 9 .
$$

Straightforward computations show the remaining cases $n=2, \ldots, 8$ of the last assertion. This concludes the proof.

In view of Theorem 3.2, the question arises whether an upper bound for $\mathcal{W}_{u}(K ; E)$ can be obtained involving $\operatorname{vol}(K)$ as the only functional of $K$. A different technique will allow us to get additional upper and lower bounds for the Wills functional just in terms of the volumes of the involved sets.

On the one hand, it is well-known that the (relative) quermassintegrals of two convex bodies satisfy the inequalities

$$
\begin{aligned}
\mathrm{W}_{i}(K ; E)^{2} \geq \mathrm{W}_{i-1}(K ; E) \mathrm{W}_{i+1}(K ; E), & 1 \leq i \leq n-1, \\
\mathrm{~W}_{i}(K ; E) \mathrm{W}_{j}(K ; E) \geq \mathrm{W}_{k}(K ; E) \mathrm{W}_{l}(K ; E), & 0 \leq k<i<j<l \leq n,
\end{aligned}
$$

which are particular cases of the Aleksandrov-Fenchel inequality (see e.g. [25, Section 7.3]). Then, we get in particular that

$$
\mathrm{W}_{i}(K ; E)^{2} \geq \mathrm{W}_{0}(K ; E) \mathrm{W}_{n}(K ; E)=\operatorname{vol}(K) \operatorname{vol}(E),
$$

and hence, for any $u \in \mathfrak{C}\left(\mathbb{R}_{\geq 0}\right)$ strictly increasing,

$$
\begin{aligned}
\mathcal{W}_{u}(K ; E) & =\sum_{i=0}^{n}\left(\begin{array}{c}
n \\
i
\end{array}\right) \mathfrak{m}_{i}^{u} \mathrm{~W}_{i}(K ; E) \\
& \geq \operatorname{vol}(K)+\mathfrak{m}_{n}^{u} \operatorname{vol}(E)+(\operatorname{vol}(K) \operatorname{vol}(E))^{1 / 2} \sum_{i=1}^{n-1}\left(\begin{array}{c}
n \\
i
\end{array}\right) \mathfrak{m}_{i}^{u} .
\end{aligned}
$$

On the other hand, we see from (3.9) that it is not possible to bound from above the Wills functional only in terms of the volumes of the involved sets, because there are convex bodies with volume arbitrarily small but having the remaining quermassintegrals bounded from below. Therefore, in order that such an upper bound for $\mathcal{W}_{u}(K ; E)$ makes sense, it is necessary to 'modify' either $K$ or $E$. In this regard we prove the following result.

Theorem 3.3. Let $u \in \mathfrak{C}\left(\mathbb{R}_{\geq 0}\right)$ be strictly increasing and such that $u(0)=0$, and let $K, E \in \mathcal{K}^{n}$ with $0 \in \operatorname{int} E$. Then there exist $T \in \operatorname{SL}(n)$ and an absolute constant $C>0$ such that

$$
\mathcal{W}_{u}(T K ; E)^{1 / n} \leq C\left(\operatorname{vol}(K)^{1 / n}+\left(\mathfrak{m}_{n}^{u}\right)^{1 / n} \operatorname{vol}(E)^{1 / n}\right)
$$


Proof. We assume without loss of generality that $0 \in K$ because the involved functionals are translation invariant. Since $f_{K, E}^{u}=e^{-u\left(|\cdot|_{E}\right)} \star \chi_{K}$ and both $e^{-u\left(|\cdot|_{E}\right)}$ and $\chi_{K}$ attain their maximums at the origin and $\left\|e^{-u\left(|\cdot|_{E}\right)}\right\|_{\infty}=$ $\left\|\chi_{K}\right\|_{\infty}=1$, Theorem $\mathrm{C}$ yields the existence of $T_{1}, T_{2} \in \mathrm{SL}(n)$ and an absolute constant $C>0$ such that

$$
\begin{aligned}
\left(\int_{\mathbb{R}^{n}} \sup _{y \in \mathbb{R}^{n}} e^{-u\left(\left|T_{1} y\right|_{E}\right)} \chi_{K}\left(T_{2} x-T_{2} y\right) \mathrm{d} x\right)^{1 / n} \\
=\left(\int_{\mathbb{R}^{n}}\left(\left(e^{-u\left(|\cdot|_{E}\right)} \circ T_{1}\right) \star\left(\chi_{K} \circ T_{2}\right)\right)(x) \mathrm{d} x\right)^{1 / n} \\
\leq C\left[\left(\int_{\mathbb{R}^{n}} e^{-u\left(\left|T_{1} x\right|_{E}\right)} \mathrm{d} x\right)^{1 / n}+\left(\int_{\mathbb{R}^{n}} \chi_{K}\left(T_{2} x\right) \mathrm{d} x\right)^{1 / n}\right]
\end{aligned}
$$

(see also Lemma 2.1). Then, writing $z=T_{1} y$ and $T=T_{1} T_{2}^{-1}$, and doing the change of variable $x=T_{1}^{-1} w$ we get, using again Lemma 2.2,

$$
\begin{aligned}
& \int_{\mathbb{R}^{n}} \sup _{y \in \mathbb{R}^{n}} e^{-u\left(\left|T_{1} y\right|_{E}\right)} \chi_{K}\left(T_{2} x-T_{2} y\right) \mathrm{d} x \\
& =\int_{\mathbb{R}^{n}} \sup _{z \in \mathbb{R}^{n}} e^{-u\left(|z|_{E}\right)} \chi_{K}\left(T_{2} T_{1}^{-1} w-T_{2} T_{1}^{-1} z\right) \mathrm{d} w \\
& =\int_{\mathbb{R}^{n}} \sup _{z \in \mathbb{R}^{n}} e^{-u\left(|z|_{E}\right)} \chi_{T_{1} T_{2}^{-1} K}(w-z) \mathrm{d} w
\end{aligned}
$$

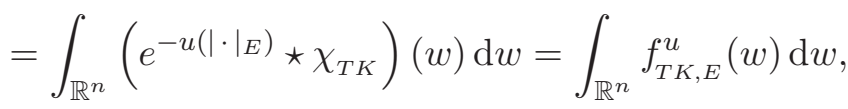

and clearly (see (3.7))

$$
\int_{\mathbb{R}^{n}} e^{-u\left(\left|T_{1} x\right|_{E}\right)} \mathrm{d} x=\int_{\mathbb{R}^{n}} e^{-u\left(|x|_{E}\right)} \mathrm{d} x=\mathfrak{m}_{n}^{u} \operatorname{vol}(E)
$$

and

$$
\int_{\mathbb{R}^{n}} \chi_{K}\left(T_{2} x\right) \mathrm{d} x=\int_{\mathbb{R}^{n}} \chi_{K}(x) \mathrm{d} x=\operatorname{vol}(K) .
$$

Altogether we obtain the result.

In the particular case of the classical Wills functional we obtain the following bounds.

Corollary 3.2. Let $K \in \mathcal{K}^{n}$ with $0 \in K$. Then there exist $T \in \operatorname{SL}(n)$ and an absolute constant $C>0$ such that

$$
1+\operatorname{vol}(K)+\left(\operatorname{vol}(K) \kappa_{n}\right)^{1 / 2} \sum_{i=1}^{n-1} \frac{\left(\begin{array}{c}
n \\
i
\end{array}\right)}{\kappa_{i}} \leq \mathcal{W}(K) \leq C\left(\operatorname{vol}\left(T^{-1} K\right)^{1 / n}+1\right)^{n} .
$$


FURTHER INEQUALITIES FOR THE (GENERALIZED) WILLS FUNCTIONAL

3.2. Relating $\mathcal{W}(\cdot)$ to other functionals. Next we reprove McMullen's result, Theorem A iv), using a different approach (see also [27] for another proof established in the setting of Gaussian processes), obtaining also a lower bound for the Wills functional in terms of the first intrinsic volume and the circumradius of the set: we show Theorem 1.2. To this end, we will use the so-called Legendre transform of a convex function, as well as some of its properties. We recall its definition.

The Legendre transform of a convex function $f: \mathbb{R}^{n} \longrightarrow \mathbb{R} \cup\{\infty\}$ is defined by

$$
\mathcal{L}(f)(x)=\sup _{y \in \mathbb{R}^{n}}(\langle x, y\rangle-f(y)),
$$

which is also a convex function (see e.g. [25, Subsection 1.6.2]). Directly related to the Legendre transform we find the polar function of a log-concave function $f: \mathbb{R}^{n} \longrightarrow \mathbb{R}_{\geq 0}$, which is defined as

$$
f^{\circ}(x)=e^{-\mathcal{L}(-\log f)(x)} .
$$

Next we prove that the Legendre transform of the Euclidean distance from a convex body is closely related to the support function of the set.

Lemma 3.1. Let $K \in \mathcal{K}^{n}$. Then

$$
\mathcal{L}\left(\pi d(\cdot, K)^{2}\right)(x)=\frac{|x|^{2}}{4 \pi}+h_{K}(x) .
$$

Proof. First we observe that, by the definition of the Legendre transform,

$$
\begin{aligned}
\mathcal{L}\left(\pi|\cdot|^{2}\right)(x) & =\sup _{y \in \mathbb{R}^{n}}\left(\langle y, x\rangle-\pi|y|^{2}\right)=\sup _{r \geq 0} \sup _{|y|=r}\left(\langle y, x\rangle-\pi|y|^{2}\right) \\
& =\sup _{r \geq 0}\left(r|x|-\pi r^{2}\right)=\frac{|x|^{2}}{4 \pi} .
\end{aligned}
$$

On the other hand,

$$
\begin{aligned}
\mathcal{L}\left(\pi d(\cdot, K)^{2}\right)(x) & =\sup _{y \in \mathbb{R}^{n}}\left(\langle y, x\rangle-\pi \inf _{z \in K}|y-z|^{2}\right)=\sup _{y \in \mathbb{R}^{n}} \sup _{z \in K}\left(\langle y, x\rangle-\pi|y-z|^{2}\right) \\
& =\sup _{z \in K} \sup _{y \in \mathbb{R}^{n}}\left(\langle y, x\rangle-\pi|y|^{2}-\pi|z|^{2}+2 \pi\langle y, z\rangle\right) \\
& =\sup _{z \in K} \sup _{y \in \mathbb{R}^{n}}\left(\langle y, x+2 \pi z\rangle-\pi|y|^{2}-\pi|z|^{2}\right) \\
& =\sup _{z \in K}\left(\mathcal{L}\left(\pi|\cdot|^{2}\right)(x+2 \pi z)-\pi|z|^{2}\right) .
\end{aligned}
$$

Then, using (3.10) we conclude the result:

$$
\begin{aligned}
\mathcal{L}\left(\pi d(\cdot, K)^{2}\right)(x) & =\sup _{z \in K}\left(\frac{|x+2 \pi z|^{2}}{4 \pi}-\pi|z|^{2}\right)=\sup _{z \in K}\left(\frac{|x|^{2}}{4 \pi}+\langle x, z\rangle\right) \\
& =\frac{|x|^{2}}{4 \pi}+h_{K}(x) .
\end{aligned}
$$

We are now in a position to prove Theorem 1.2. 
Proof of Theorem 1.2. First we note that since all the involved functionals, namely, $\mathrm{V}_{1}(K), \mathrm{R}(K)$ and $\mathcal{W}(K)$, are invariant under translations, we may assume that the origin is the circumcenter of $K$, i.e., that $K \subset \mathrm{R}(K) B_{2}^{n}$. Then $|y| \leq \mathrm{R}(K)$ for all $y \in K$.

We start showing the lower bound. For any convex body $K \in \mathcal{K}^{n}$ we have

$$
\begin{aligned}
\mathcal{W}(K) & =\int_{\mathbb{R}^{n}} e^{-\pi d(x, K)^{2}} \mathrm{~d} x=\int_{\mathbb{R}^{n}} e^{-\pi \inf _{y \in K}|x-y|^{2}} \mathrm{~d} x \\
& =\int_{\mathbb{R}^{n}} e^{-\pi|x|^{2}} e^{\pi \sup _{y \in K}\left(2\langle x, y\rangle-|y|^{2}\right)} \mathrm{d} x,
\end{aligned}
$$

and doing the change of variable $\sqrt{2 \pi} x=z$ we get

$$
\begin{aligned}
\mathcal{W}(K) & =\int_{\mathbb{R}^{n}} \frac{e^{-\frac{|z|^{2}}{2}}}{(2 \pi)^{n / 2}} e^{\sup _{y \in K}\left(\sqrt{2 \pi}\langle z, y\rangle-\pi|y|^{2}\right)} \mathrm{d} z \\
& =\int_{\mathbb{R}^{n}} e^{\sup _{y \in K}\left(\sqrt{2 \pi}\langle z, y\rangle-\pi|y|^{2}\right)} \mathrm{d} \gamma_{n}(z) .
\end{aligned}
$$

Then

$$
\begin{aligned}
\mathcal{W}(K) & \geq \int_{\mathbb{R}^{n}} e^{\sup _{y \in K}\left(\sqrt{2 \pi}\langle z, y\rangle-\pi \mathrm{R}(K)^{2}\right)} \mathrm{d} \gamma_{n}(z) \\
& =e^{-\pi \mathrm{R}(K)^{2}} \int_{\mathbb{R}^{n}} e^{\sqrt{2 \pi} \sup _{y \in K}\langle z, y\rangle} \mathrm{d} \gamma_{n}(z)=e^{-\pi \mathrm{R}(K)^{2}} \int_{\mathbb{R}^{n}} e^{\sqrt{2 \pi} h_{K}(z)} \mathrm{d} \gamma_{n}(z),
\end{aligned}
$$

and Jensen's inequality (see e.g. [25, page 20]) applied to the convex function $e^{x}$ yields

$$
\mathcal{W}(K) \geq e^{-\pi \mathrm{R}(K)^{2}} e^{\sqrt{2 \pi} \int_{\mathbb{R}^{n}} h_{K}(z) \mathrm{d} \gamma_{n}(z)} .
$$

So it remains to compute the above integral in the exponential function:

$$
\begin{aligned}
\int_{\mathbb{R}^{n}} h_{K}(z) \mathrm{d} \gamma_{n}(z) & =\frac{1}{(2 \pi)^{n / 2}} \int_{\mathbb{R}^{n}} e^{-\frac{|z|^{2}}{2}} h_{K}(z) \mathrm{d} z \\
& =\frac{1}{(2 \pi)^{n / 2}} \int_{\mathbb{S}^{n-1}} \int_{0}^{\infty} r^{n} e^{-\frac{r^{2}}{2}} h_{K}(u) \mathrm{d} r \mathrm{~d} u \\
& =\frac{1}{(2 \pi)^{n / 2}} \int_{\mathbb{S}^{n-1}} h_{K}(u)\left(\int_{0}^{\infty} r^{n} e^{-\frac{r^{2}}{2}} \mathrm{~d} r\right) \mathrm{d} u
\end{aligned}
$$

and with the change of variable $r^{2} / 2=t$ and using (1.2) we get

$$
\int_{\mathbb{R}^{n}} h_{K}(z) \mathrm{d} \gamma_{n}(z)=\frac{2^{(n-1) / 2}}{(2 \pi)^{n / 2}} \Gamma\left(\frac{n-1}{2}+1\right) \kappa_{n-1} \mathrm{~V}_{1}(K)=\frac{1}{\sqrt{2 \pi}} \mathrm{V}_{1}(K) .
$$

Therefore, we conclude that

$$
\mathcal{W}(K) \geq e^{-\pi \mathrm{R}(K)^{2}} e^{\sqrt{2 \pi} \frac{1}{\sqrt{2 \pi}} \mathrm{V}_{1}(K)}=e^{\mathrm{V}_{1}(K)-\pi \mathrm{R}(K)^{2}} .
$$

To prove the upper bound we note again that, since $\mathrm{V}_{1}(K)$ and $\mathcal{W}(K)$ are invariant under translations, we may assume that $0 \in K$. We also observe 
that, by Lemma 3.1,

$$
\left(f_{K, B_{2}^{n}}^{\pi(\cdot)^{2}}(x)=\left(e^{-\pi d(\cdot, K)^{2}}\right)^{\circ}(x)=e^{-\mathcal{L}\left(\pi d(\cdot, K)^{2}\right)(x)}=e^{-\frac{|x|^{2}}{4 \pi}-h_{K}(x)},\right.
$$

and so, doing the change of variable $x=\sqrt{2 \pi} z$ and using Jensen's inequality and (3.11) we get

$$
\begin{aligned}
\int_{\mathbb{R}^{n}}\left(f_{K, B_{2}^{n}}^{\pi(\cdot)^{2}}\right)^{\circ}(x) \mathrm{d} x & =\int_{\mathbb{R}^{n}} e^{-\frac{|x|^{2}}{4 \pi}} e^{-h_{K}(x)} \mathrm{d} x=(2 \pi)^{n / 2} \int_{\mathbb{R}^{n}} e^{-\frac{|z|^{2}}{2}} e^{-\sqrt{2 \pi} h_{K}(z)} \mathrm{d} z \\
& =(2 \pi)^{n} \int_{\mathbb{R}^{n}} e^{-\sqrt{2 \pi} h_{K}(z)} \mathrm{d} \gamma_{n}(z) \\
& \geq(2 \pi)^{n} e^{-\sqrt{2 \pi} \int_{\mathbb{R}^{n}} h_{K}(z) \mathrm{d} \gamma_{n}(z)}=(2 \pi)^{n} e^{-\mathrm{V}_{1}(K)} .
\end{aligned}
$$

Now, since $0 \in K$, then $\left\|f_{K, B_{2}^{n}}^{\pi\left(\cdot \|^{2}\right.}\right\|_{\infty}=f_{K, B_{2}^{n}}^{\pi(\cdot)^{2}}(0)=1$, and hence we can apply the functional version of Blaschke-Santaló's inequality ([20, Theorems 1.1 and 1.2, and Remark (2) in page 181)]; see also [6, Theorem 1.3]), namely,

$$
(2 \pi)^{n} \geq \int_{\mathbb{R}^{n}} f_{K, B_{2}^{n}}^{\pi(\cdot)^{2}}(x) \mathrm{d} x \int_{\mathbb{R}^{n}}\left(f_{K, B_{2}^{n}}^{\pi(\cdot)^{2}}\right)^{\circ}(x) \mathrm{d} x,
$$

to obtain

$$
(2 \pi)^{n} \geq \mathcal{W}(K) \int_{\mathbb{R}^{n}}\left(f_{K, B_{2}^{n}}^{\pi(\cdot)^{2}}\right)^{\circ}(x) \mathrm{d} x \geq \mathcal{W}(K)(2 \pi)^{n} e^{-\mathrm{V}_{1}(K)},
$$

which finishes the proof.

3.3. On 0-symmetric convex bodies in John position. It is well-known ([7, Theorem 3]) that the cube $[-1,1]^{n}$ maximizes the volume functional among all 0 -symmetric convex bodies in John position (see also [25, Theorem 10.13.2] and the references therein). The same property also holds for the mean width (see e.g. [25, page 602$]): \mathrm{b}(K) \leq \mathrm{b}\left([-1,1]^{n}\right)$ for every 0 symmetric $K \in \mathcal{K}^{n}$ which is in John position. Nevertheless, to the best of the authors' knowledge, similar results for the remaining intrinsic volumes are not known. Here, following Ball's idea on the application of the BrascampLieb inequality to data provided by the conditions in John's theorem, we show that the cube $[-1,1]^{n}$ also maximizes the Wills functional among all 0-symmetric convex bodies in John position, i.e., we prove Theorem 1.3.

Proof of Theorem 1.3. Since $K$ is in John position, there exist $c_{1}, \ldots, c_{m}>0$ and vectors $v_{1}, \ldots, v_{m} \in \mathbb{S}^{n-1} \cap \mathrm{bd} K, n \leq m \leq n(n+1) / 2$, such that the identity matrix $\mathrm{I}_{n}=\sum_{i=1}^{m} c_{i} v_{i} \otimes v_{i}$ (see [25, Theorem 10.12.1]); here $v_{j} \otimes v_{j}$ is the projection in the direction of $v_{j}$, i.e., $\left(v_{j} \otimes v_{j}\right)(x)=\left\langle x, v_{j}\right\rangle v_{j}$. In particular, $\sum_{i=1}^{m} c_{i}=n$. From $B_{2}^{n} \subset K$ and $v_{i} \in \mathbb{S}^{n-1} \cap \mathrm{bd} K$ for $i=1, \ldots, m$, we get $K \subset\left\{x \in \mathbb{R}^{n}:\left\langle x, v_{i}\right\rangle \leq 1\right\}$ and thus, since $K$ is 0 -symmetric, we have $K \subset L=\left\{x \in \mathbb{R}^{n}:\left|\left\langle x, v_{i}\right\rangle\right| \leq 1\right\}$. This implies that $\mathcal{W}(K) \leq \mathcal{W}(L)$.

As usual in the literature, we write $\mathrm{e}_{i}$ to represent the $i$-th canonical unit vector. Let $\operatorname{span}\{v\}$ denote the 1-dimensional linear subspace spanned by 
the vector $v \in \mathbb{R}^{n}$, and let $f_{i}(t)=e^{-\pi d\left(t v_{i}, P_{\mathrm{span}\left\{v_{i}\right\}} L\right)^{2}}, t \in \mathbb{R}$, for $i=1, \ldots, m$. Using that $\sum_{i=1}^{m} c_{i}=n$ together with Theorem A i) and the fact that $\mathcal{W}([-v, v])=\mathcal{W}\left(\left[-\mathrm{e}_{i}, \mathrm{e}_{i}\right]\right)$ for all $v \in \mathbb{S}^{n-1}$ and all $i=1, \ldots, n$, one gets

$\mathcal{W}\left([-1,1]^{n}\right)=\prod_{i=1}^{n} \mathcal{W}\left(\left[-\mathrm{e}_{i}, \mathrm{e}_{i}\right]\right)=\prod_{i=1}^{m} \mathcal{W}\left(P_{\operatorname{span}\left\{v_{i}\right\}} L\right)^{c_{i}}=\prod_{i=1}^{m}\left(\int_{\mathbb{R}} f_{i}(t) \mathrm{d} t\right)^{c_{i}}$,

and the geometric Brascamp-Lieb inequality (see [25, Theorem 10.13.1] and the references therein) gives

$$
\begin{aligned}
\mathcal{W}\left([-1,1]^{n}\right)=\prod_{i=1}^{m}\left(\int_{\mathbb{R}} f_{i}(t) \mathrm{d} t\right)^{c_{i}} & \geq \int_{\mathbb{R}^{n}} \prod_{i=1}^{m} f_{i}\left(\left\langle x, v_{i}\right\rangle\right)^{c_{i}} \mathrm{~d} x \\
& =\int_{\mathbb{R}^{n}} e^{-\pi \sum_{i=1}^{m} c_{i} d\left(\left\langle x, v_{i}\right\rangle v_{i}, P_{\mathrm{span}\left\{v_{i}\right\}} L\right)^{2}} \mathrm{~d} x .
\end{aligned}
$$

Thus, to conclude the proof, it is enough to show that

$$
\sum_{i=1}^{m} c_{i} d\left(\left\langle x, v_{i}\right\rangle v_{i}, P_{\operatorname{span}\left\{v_{i}\right\}} L\right)^{2} \leq d(x, L)^{2}
$$

because, in that case, we have that

$$
\int_{\mathbb{R}^{n}} e^{-\pi \sum_{i=1}^{m} c_{i} d\left(\left\langle x, v_{i}\right\rangle v_{i}, P_{\text {span }\left\{v_{i}\right\}} L\right)^{2}} \mathrm{~d} x \geq \int_{\mathbb{R}^{n}} e^{-\pi d(x, L)^{2} \mathrm{~d} x}=\mathcal{W}(L) \geq \mathcal{W}(K) .
$$

To this end we notice that, for any given $x_{0} \in L$, all $x \in \mathbb{R}^{n}$ and any $i=1, \ldots, m$, we have

$$
d\left(\left\langle x, v_{i}\right\rangle v_{i}, P_{\operatorname{span}\left\{v_{i}\right\}} L\right)^{2} \leq d\left(\left\langle x, v_{i}\right\rangle v_{i},\left\langle x_{0}, v_{i}\right\rangle v_{i}\right)^{2}=\left\langle x-x_{0}, v_{i}\right\rangle^{2}
$$

and thus, using that $\mathrm{I}_{n}=\sum_{i=1}^{m} c_{i} v_{i} \otimes v_{i}$,

$$
\sum_{i=1}^{m} c_{i} d\left(\left\langle x, v_{i}\right\rangle v_{i}, P_{\mathrm{span}\left\{v_{i}\right\}} L\right)^{2} \leq \sum_{i=1}^{m} c_{i}\left\langle x-x_{0}, v_{i}\right\rangle^{2}=\left|x-x_{0}\right|^{2} .
$$

In particular $\sum_{i=1}^{m} c_{i} d\left(\left\langle x, v_{i}\right\rangle v_{i}, P_{\text {span }\left\{v_{i}\right\}} L\right)^{2} \leq d(x, L)^{2}$, as desired.

\section{Brunn-Minkowski type inequalities for the Wills functional}

Relating the volume with the Minkowski addition of convex bodies, one is led to the famous Brunn-Minkowski inequality. One form of it states that if $K, L \in \mathcal{K}^{n}$ are convex bodies, and $\lambda \in(0,1)$, then

$$
\operatorname{vol}((1-\lambda) K+\lambda L)^{1 / n} \geq(1-\lambda) \operatorname{vol}(K)^{1 / n}+\lambda \operatorname{vol}(L)^{1 / n}
$$

with equality, if $\operatorname{vol}(K) \operatorname{vol}(L)>0$, if and only if $K$ and $L$ are homothetic. In other words, the volume functional $\operatorname{vol}(\cdot)$ is $(1 / n)$-concave.

The above inequality admits a generalization in the context of intrinsic volumes. Indeed, $\mathrm{V}_{i}(\cdot)$ is a $(1 / i)$-concave functional for all $i=1, \ldots, n$ (see e.g. [25, Theorem 7.4.5]), namely

$$
\mathrm{V}_{i}((1-\lambda) K+\lambda L)^{1 / i} \geq(1-\lambda) \mathrm{V}_{i}(K)^{1 / i}+\lambda \mathrm{V}_{i}(L)^{1 / i}
$$


for any $K, L \in \mathcal{K}^{n}$ and all $\lambda \in(0,1)$. In particular, $\mathrm{V}_{i}(\cdot)$ is $(1 / n)$-concave for all $i=0, \ldots, n$ (we recall that $\mathrm{V}_{0}(K)=1$ for any $K \in \mathcal{K}^{n}$ ) and thus one might expect that the same holds for the Wills functional $\mathcal{W}(\cdot)=\sum_{i=0}^{n} \mathrm{~V}_{i}(\cdot)$. In general, this is not the case, as the following example shows.

Example 4.1. The $i$-th intrinsic volume of the Euclidean ball is $\mathrm{V}_{i}\left(r B_{2}^{n}\right)=$ $\left(\begin{array}{c}n \\ i\end{array}\right) / \kappa_{n-i} \mathrm{~W}_{n-i}\left(r B_{2}^{n}\right)=\left(\begin{array}{c}n \\ i\end{array}\right) \kappa_{n} / \kappa_{n-i} r^{i}$. Then, one can see that the inequality

$$
\mathcal{W}\left(\frac{r+R}{2} B_{2}^{n}\right)^{1 / n} \geq \frac{1}{2} \mathcal{W}\left(r B_{2}^{n}\right)^{1 / n}+\frac{1}{2} \mathcal{W}\left(R B_{2}^{n}\right)^{1 / n}
$$

is, in general, not true, just taking $r=1, R=2$ and $n=2,3,4 \ldots$

The Euclidean balls $B_{2}^{n}$ and $2 B_{2}^{n}$ also show that the additive version of the Brunn-Minkowski inequality for the Wills functional, namely,

$$
\mathcal{W}\left(B_{2}^{n}+2 B_{2}^{n}\right)^{1 / n} \geq \mathcal{W}\left(B_{2}^{n}\right)^{1 / n}+\mathcal{W}\left(2 B_{2}^{n}\right)^{1 / n}
$$

is, in general, not true.

Although, as seen, the Wills functional is not a $(1 / n)$-concave functional, it is not "far" from being so. Indeed, when dealing with $K$ and $L$ orthogonal boxes we have $\mathcal{W}((1-\lambda) K+\lambda L)^{1 / n} \geq(1-\lambda) \mathcal{W}(K)^{1 / n}+\lambda \mathcal{W}(L)^{1 / n}$ : this can be shown as a direct consequence of Theorem A i), using the arithmeticgeometric mean inequality, and the fact that the Wills functional of a segment $\ell$ is $\mathcal{W}(\ell)=1+\operatorname{vol}_{1}(\ell)$. Moreover, by adding the additional constant $1 /(n !)^{1 / n}$ on the right-hand side of the above inequality, it becomes true for arbitrary convex bodies, as Theorem 1.5 shows. We notice that this constant is of the order of $e / n$. We state and prove it in the more general setting of the generalized Wills functional.

Theorem 4.1. Let $K, L, E \in \mathcal{K}^{n}$ with $0 \in \operatorname{int} E, \lambda \in(0,1)$ and $u \in \mathfrak{C}\left(\mathbb{R}_{\geq 0}\right)$ strictly increasing. Then

$\mathcal{W}_{u}((1-\lambda) K+\lambda L ; E)^{1 / n} \geq \frac{e^{-\frac{n-1}{n} u(0)}}{(n !)^{1 / n}}\left((1-\lambda) \mathcal{W}_{u}(K ; E)^{1 / n}+\lambda \mathcal{W}_{u}(L ; E)^{1 / n}\right)$

Proof. For the sake of brevity we set $M_{\lambda}=(1-\lambda) K+\lambda L$. The BrunnMinkowski inequality (4.1) implies that

$\operatorname{vol}\left(M_{\lambda}+u^{-1}(s) E\right)^{1 / n} \geq(1-\lambda) \operatorname{vol}\left(K+u^{-1}(s) E\right)^{1 / n}+\lambda \operatorname{vol}\left(L+u^{-1}(s) E\right)^{1 / n}$ for all $s \geq u(0)$, and then we clearly get

$$
\begin{aligned}
\int_{u(0)}^{\infty} \operatorname{vol}\left(M_{\lambda}+u^{-1}(s) E\right)^{1 / n} e^{-s} \mathrm{~d} s \geq & (1-\lambda) \int_{u(0)}^{\infty} \operatorname{vol}\left(K+u^{-1}(s) E\right)^{1 / n} e^{-s} \mathrm{~d} s \\
& +\lambda \int_{u(0)}^{\infty} \operatorname{vol}\left(L+u^{-1}(s) E\right)^{1 / n} e^{-s} \mathrm{~d} s .
\end{aligned}
$$


On one hand, by Jensen's inequality, together with (2.3), we have

$$
\begin{aligned}
\int_{u(0)}^{\infty} \operatorname{vol}\left(M_{\lambda}+u^{-1}(s) E\right)^{1 / n} e^{-s} \mathrm{~d} s & \leq\left(\int_{u(0)}^{\infty} \operatorname{vol}\left(M_{\lambda}+u^{-1}(s) E\right) e^{-s} \mathrm{~d} s\right)^{1 / n} \\
& =\mathcal{W}_{u}\left(M_{\lambda} ; E\right)^{1 / n}
\end{aligned}
$$

on the other hand, doing the change of variable $t=s-u(0)$, we can apply the extension of Berwald's inequality (cf. [8, Satz 8]) which is proved in [3, Lemma 2.1], and with (2.3) we obtain

$$
\begin{aligned}
\int_{u(0)}^{\infty} \operatorname{vol}\left(K+u^{-1}(s) E\right)^{1 / n} e^{-s} \mathrm{~d} s & \geq \frac{e^{-\frac{n-1}{n} u(0)}}{(n !)^{1 / n}}\left(\int_{u(0)}^{\infty} \operatorname{vol}\left(K+u^{-1}(s) E\right) e^{-s} \mathrm{~d} s\right)^{1 / n} \\
& =\frac{e^{-\frac{n-1}{n} u(0)}}{(n !)^{1 / n}} \mathcal{W}_{u}(K ; E)^{1 / n} .
\end{aligned}
$$

The same holds for $L$ in place of $K$. Altogether, from (4.3), (4.4) and (4.5), we get (4.2).

In the case of the classical Wills functional, from (4.2) for $u=\pi(\cdot)^{2}$ and $E=B_{2}^{n}$, we get Theorem 1.5:

$$
\mathcal{W}((1-\lambda) K+\lambda L)^{1 / n} \geq \frac{1}{(n !)^{1 / n}}\left((1-\lambda) \mathcal{W}(K)^{1 / n}+\lambda \mathcal{W}(L)^{1 / n}\right) .
$$

Aiming to get a "real" concavity property for the Wills functional, we will exploit its integral formula via a log-concave function (cf. (2.3)) as well as the corresponding machinery: the Prékopa-Leindler inequality (Theorem B for $p=0)$. This gives rise to Theorem 1.4. Again we state and prove it in the general setting: we see that the generalized Wills functional is log-concave.

Theorem 4.2. Let $E \in \mathcal{K}^{n}$ with $0 \in \operatorname{int} E$ and let $u \in \mathfrak{C}\left(\mathbb{R}_{\geq 0}\right)$. Then $\mathcal{W}_{u}(\cdot ; E)$ is log-concave, i.e., for any $K, L \in \mathcal{K}^{n}$ and all $\lambda \in(0, \overline{1})$,

$$
\mathcal{W}_{u}((1-\lambda) K+\lambda L ; E) \geq \mathcal{W}_{u}(K ; E)^{1-\lambda} \mathcal{W}_{u}(L ; E)^{\lambda} \text {. }
$$

Proof. By the triangle inequality for $|\cdot|_{E}$ we have that

$$
d_{E}((1-\lambda) x+\lambda y,(1-\lambda) K+\lambda L) \leq(1-\lambda) d_{E}(x, K)+\lambda d_{E}(y, L)
$$

for every $x, y \in \mathbb{R}^{n}$, and hence, from the convexity and monotonicity of $u$,

$u\left(d_{E}((1-\lambda) x+\lambda y,(1-\lambda) K+\lambda L)\right) \leq(1-\lambda) u\left(d_{E}(x, K)\right)+\lambda u\left(d_{E}(y, L)\right)$.

Therefore, the functions $f=f_{K, E}^{u}, g=f_{L, E}^{u}$ and $h=f_{(1-\lambda) K+\lambda L, E}^{u}$ are in the conditions of the Prékopa-Leindler inequality (Theorem B for $p=0$ ), and thus (2.6) yields (4.6). 
Remark 4.1. Under the assumption that the involved convex bodies have a common projection onto a hyperplane, the concavity of the Wills functional can be improved. More precisely, if $K, L \in \mathcal{K}^{n}$ are convex bodies with nonempty interior such that $P_{H} K=P_{H} L$ for some hyperplane $H \in \mathrm{G}(n, n-1)$ then, for any $E \in \mathcal{K}^{n}$ with $0 \in \operatorname{int} E$, all $\lambda \in(0,1)$ and any $u \in \mathfrak{C}\left(\mathbb{R}_{\geq 0}\right)$,

$$
\mathcal{W}_{u}((1-\lambda) K+\lambda L ; E) \geq(1-\lambda) \mathcal{W}_{u}(K ; E)+\lambda \mathcal{W}_{u}(L ; E) .
$$

Indeed, this follows from the concavity of the relative quermassintegrals in this setting (see e.g. [25, Theorem 7.7.2]) jointly with Proposition 2.1, namely, $\mathcal{W}_{u}(\cdot ; E)=\sum_{i=0}^{n}\left(\begin{array}{c}n \\ i\end{array}\right) \mathfrak{m}_{i}^{u} \mathrm{~W}_{i}(\cdot ; E)$.

Coming back to the classical Wills functional, from (4.6) for $u=\pi(\cdot)^{2}$ and $E=B_{2}^{n}$, we have Theorem 1.4:

$$
\mathcal{W}((1-\lambda) K+\lambda L) \geq \mathcal{W}(K)^{1-\lambda} \mathcal{W}(L)^{\lambda} .
$$

Without any extra assumption on the convex bodies (cf. Remark 4.1), this (log-) concavity seems not possible to be improved: considering again the Wills functionals of Euclidean balls (see Example 4.1), numerical computations show that for the balls $0.2 B_{2}^{n}$ and $0.05 B_{2}^{n}$ (with $\lambda=1 / 2$ ), the $(1 /(n+1))$-concavity does not hold in general for $n>2$, i.e.,

$\mathcal{W}\left(\frac{0.25}{2} B_{2}^{n}\right)^{1 /(n+1)}<\frac{1}{2} \mathcal{W}\left(0.2 B_{2}^{n}\right)^{1 /(n+1)}+\frac{1}{2} \mathcal{W}\left(0.05 B_{2}^{n}\right)^{1 /(n+1)}$ for $n>2 ;$

moreover, these numerical calculations for the previous balls suggest that for any $p>3$, there exists a value of the dimension $n<p$ such that the $(1 / p)$-concavity does not hold.

However, dimension $n=2$ is a singular case: here, the classical Wills functional is $(1 /(n+1))$-concave; we recall that, even in the planar case, the Wills functional is not $(1 / n)$-concave, as mentioned in Example 4.1. This is the content of the following result, in which we exploit a suitable generalized Wills functional to derive additional information for the classical one.

Theorem 4.3. Let $K, L \in \mathcal{K}^{2}$ and $\lambda \in(0,1)$. Then

$$
\mathcal{W}((1-\lambda) K+\lambda L)^{1 / 3} \geq(1-\lambda) \mathcal{W}(K)^{1 / 3}+\lambda \mathcal{W}(L)^{1 / 3} .
$$

Proof. First we assume that there exists an increasing non-negative continuous function $\phi:[0, a] \longrightarrow \mathbb{R}_{\geq 0}$, for some $a>0$, such that

$$
\int_{0}^{a} \phi(t) t^{i} \mathrm{~d} t=\frac{1}{\kappa_{i}}, \quad \text { for } i=0,1,2 .
$$

Then, the function $G: \mathbb{R}_{\geq 0} \longrightarrow \mathbb{R}_{\geq 0}$ defined by $G(t)=\mu([t, \infty))$, where $\mu$ is the measure on $\mathbb{R}_{\geq 0}$, concentrated on $[0, a]$, given by $\mathrm{d} \mu(t)=\phi(t) \mathrm{d} t$, satisfies that (see [18, Lemma 1.1] and the references therein)

$$
\int_{\mathbb{R}^{2}} G(d(x, M)) \mathrm{d} x=\mathcal{W}(M)
$$


for any convex body $M \in \mathcal{K}^{2}$. We consider the functions $f=G(d(\cdot, K))$, $g=G(d(\cdot, L))$ and $h=G(d(\cdot,(1-\lambda) K+\lambda L))$. By the fundamental theorem of calculus, the derivative of $G$ fulfils $G^{\prime}(t)=-\phi(t)$ for all $t \in[0, a]$, which, from the monotonicity of $\phi$, implies that $G$ is concave on $[0, a]$. This, together with (4.7) for $E=B_{2}^{n}$ and the fact that $G$ is a decreasing function, yields $h((1-\lambda) x+\lambda y) \geq(1-\lambda) f(x)+\lambda g(y)$ for all $x, y \in \mathbb{R}^{2}$ such that $f(x) g(y)>0$. Thus, by the Borell-Brascamp-Lieb inequality (Theorem B), we get that

$$
\begin{aligned}
\int_{\mathbb{R}^{2}} G(d(x,(1-\lambda) K+\lambda L)) \mathrm{d} x & \\
\geq & \geq\left[(1-\lambda)\left(\int_{\mathbb{R}^{2}} G(d(x, K)) \mathrm{d} x\right)^{1 / 3}+\lambda\left(\int_{\mathbb{R}^{2}} G(d(x, L)) \mathrm{d} x\right)^{1 / 3}\right]^{3},
\end{aligned}
$$

which, using (4.10), gives (4.8).

To conclude we prove the existence of such a function $\phi$. We define it by $\phi(t)=a_{1}+a_{2} t+a_{3} t^{2}$, for suitable $a_{1}, a_{2}, a_{3} \in \mathbb{R}$ to be determined later on. Then, conditions (4.9) yield the system of linear equations in $\left(a_{1}, a_{2}, a_{3}\right)$

$$
\left\{\begin{aligned}
1 & =\int_{0}^{a} \phi(t) \mathrm{d} t=a_{1} a+\frac{a_{2} a^{2}}{2}+\frac{a_{3} a^{3}}{3} \\
\frac{1}{2} & =\int_{0}^{a} \phi(t) t \mathrm{~d} t=\frac{a_{1} a^{2}}{2}+\frac{a_{2} a^{3}}{3}+\frac{a_{3} a^{4}}{4} \\
\frac{1}{\pi} & =\int_{0}^{a} \phi(t) t^{2} \mathrm{~d} t=\frac{a_{1} a^{3}}{3}+\frac{a_{2} a^{4}}{4}+\frac{a_{3} a^{5}}{5}
\end{aligned}\right.
$$

whose solution is

$$
\begin{aligned}
& a_{1}=\frac{9 \pi a^{2}-18 \pi a+30}{\pi a^{3}}, \quad a_{2}=\frac{-36 \pi a^{2}+96 \pi a-180}{\pi a^{4}}, \\
& a_{3}=\frac{30 \pi a^{2}-90 \pi a+180}{\pi a^{5}} .
\end{aligned}
$$

By studying the sign of the above quadratic polynomials in the variable $a$, one finds we can choose an appropriate value of $a$, for instance $a=0.91$, so that $a_{i}>0$ for all $i=1,2,3$; therefore, $\phi$ is non-negative and increasing on $[0, a]$. This finishes the proof.

We conclude this section by showing that, although the additive version of the Brunn-Minkowski inequality does not hold for the Wills functional (cf. Example 4.1), $\mathcal{W}(\cdot)$ satisfies a reverse Brunn-Minkowski inequality with exponent $1 / n$ : we prove Theorem 1.6. Indeed, we state and show it in the more general setting of the generalized Wills functional.

Theorem 4.4. Let $K, L, E \in \mathcal{K}^{n}$ with $0 \in \operatorname{int} E$ and let $u \in \mathfrak{C}\left(\mathbb{R}_{\geq 0}\right)$ with $u(0)=0$. Then there exist $T \in \mathrm{SL}(n)$ and an absolute constant $C>0$ such that

$$
\mathcal{W}_{u}(K+T L ; E)^{1 / n} \leq C\left[\mathcal{W}_{u}(K ; E)^{1 / n}+\mathcal{W}_{u}(L ; E)^{1 / n}\right]
$$


Proof. We may assume without loss of generality that $0 \in K \cap L$ because the involved functionals are invariant under translations.

Since $\left\|f_{K, E}^{u}\right\|_{\infty}=\left\|f_{L, E}^{u}\right\|_{\infty}=f_{K, E}^{u}(0)=f_{L, E}^{u}(0)=1$, Theorem $\mathrm{C}$ yields the existence of $T_{1}, T_{2} \in \mathrm{SL}(n)$ and an absolute constant $C>0$ such that

$$
\begin{aligned}
& \left(\int_{\mathbb{R}^{n}}\left(\left(f_{K, E}^{u} \circ T_{1}\right) \star\left(f_{L, E}^{u} \circ T_{2}\right)\right)(x) \mathrm{d} x\right)^{1 / n} \\
& \quad \leq C\left[\left(\int_{\mathbb{R}^{n}}\left(f_{K, E}^{u} \circ T_{1}\right)(x) \mathrm{d} x\right)^{1 / n}+\left(\int_{\mathbb{R}^{n}}\left(f_{L, E}^{u} \circ T_{2}\right)(x) \mathrm{d} x\right)^{1 / n}\right] .
\end{aligned}
$$

On one hand, denoting by $f=f_{K, E}^{u} \circ T_{1}$ and $g=f_{L, E}^{u} \circ T_{2}$, we get

$$
\int_{\mathbb{R}^{n}} f(x) \mathrm{d} x=\int_{\mathbb{R}^{n}} e^{-u\left(d_{E}\left(T_{1} x, K\right)\right)} \mathrm{d} x=\int_{\mathbb{R}^{n}} e^{-u\left(d_{E}(x, K)\right)} \mathrm{d} x=\mathcal{W}_{u}(K ; E)
$$

and, analogously,

$$
\int_{\mathbb{R}^{n}} g(x) \mathrm{d} x=\mathcal{W}_{u}(L ; E)
$$

On the other hand, writing $T=T_{2} T_{1}^{-1}$ and using Lemma 2.1 and Lemma 2.2, we have

$$
\begin{aligned}
\int_{\mathbb{R}^{n}}(f \star g)(x) \mathrm{d} x & =\int_{\mathbb{R}^{n}}\left(f_{K, E}^{u} \star\left(f_{L, E}^{u} \circ T\right)\right)\left(T_{1}(x)\right) \mathrm{d} x \\
& =\int_{\mathbb{R}^{n}}\left(f_{K, E}^{u} \star\left(f_{L, E}^{u} \circ T\right)\right)(x) \mathrm{d} x \\
& =\int_{\mathbb{R}^{n}}\left(f_{K, E}^{u} \star\left(\left(e^{-u\left(|\cdot|_{E}\right)} \circ T\right) \star\left(\chi_{L} \circ T\right)\right)\right)(x) \mathrm{d} x \\
& =\int_{\mathbb{R}^{n}}\left(\left(e^{-u\left(|\cdot|_{E}\right)} \star \chi_{K}\right) \star\left(\left(e^{-u\left(|\cdot|_{E}\right)} \circ T\right) \star \chi_{T^{-1} L}\right)\right)(x) \mathrm{d} x .
\end{aligned}
$$

Now, since

$$
\left(e^{-u\left(|\cdot|_{E}\right)} \star e^{-u\left(|T(\cdot)|_{E}\right)}\right)(x) \geq e^{-u\left(|x|_{E}\right)} e^{-u\left(|T(0)|_{E}\right)}=e^{-u\left(|x|_{E}\right)},
$$

we have, from Lemma 2.2, that

$$
\begin{aligned}
\left(e^{-u\left(|\cdot|_{E}\right)} \star \chi_{K}\right) \star\left(\left(e^{-u\left(|\cdot|_{E}\right)} \circ T\right) \star \chi_{T^{-1} L}\right) & =e^{-u\left(|\cdot|_{E}\right)} \star e^{-u\left(|T(\cdot)|_{E}\right)} \star \chi_{K+T^{-1} L} \\
& \geq e^{-u\left(|\cdot|_{E}\right)} \star \chi_{K+T^{-1} L}=f_{K+T^{-1} L, E}^{u},
\end{aligned}
$$

and so we get

$$
\int_{\mathbb{R}^{n}}(f \star g)(x) \mathrm{d} x \geq \int_{\mathbb{R}^{n}} f_{K+T^{-1} L, E}^{u}(x) \mathrm{d} x=\mathcal{W}_{u}\left(K+T^{-1} L ; E\right) .
$$

Altogether concludes the proof. 


\section{Rogers-Shephard type inequalities For the Wills Functional}

In this last section we obtain Rogers-Shephard type inequalities for the Wills functional. First we study section/projection Rogers-Shephard type relations.

5.1. The Wills functional for projections and sections. We start proving Theorem 1.7, for which we use the following result that we state in the general setting of the generalized Wills functional.

Proposition 5.1. Let $K, E \in \mathcal{K}^{n}$ with $0 \in \operatorname{int} E$, let $u \in \mathfrak{C}\left(\mathbb{R}_{\geq 0}\right)$ and $H \in \mathrm{G}(n, k)$. Then

$$
\mathcal{W}_{u}^{(k)}\left(P_{H} K ; P_{H} E\right) \mathcal{W}_{u}^{(n-k)}\left(K \cap H^{\perp} ; E \cap H^{\perp}\right) \leq\left(\begin{array}{l}
n \\
k
\end{array}\right) e^{-u(0)} \mathcal{W}_{u}(K ; E) .
$$

Proof. Using Lemma 2.3 and applying (2.9) to $f_{K, E}^{u}$ we get

$$
\begin{aligned}
\mathcal{W}_{u}^{(k)}\left(P_{H} K ; P_{H} E\right) & \mathcal{W}_{u}^{(n-k)}\left(K \cap H^{\perp} ; E \cap H^{\perp}\right) \\
& =\int_{H} f_{P_{H} K, P_{H} E}^{u}(x) \mathrm{d} x \int_{H^{\perp}} f_{K \cap H^{\perp}, E \cap H^{\perp}}^{u}(y) \mathrm{d} y \\
& \leq \int_{H}\left(P_{H} f_{K, E}^{u}\right)(x) \mathrm{d} x \int_{H^{\perp}} f_{K, E}^{u}(y) \mathrm{d} y \\
& \leq\left(\begin{array}{c}
n \\
k
\end{array}\right)\left\|f_{K, E}^{u}\right\|_{\infty} \int_{\mathbb{R}^{n}} f_{K, E}^{u}(z) \mathrm{d} z=\left(\begin{array}{l}
n \\
k
\end{array}\right) e^{-u(0)} \mathcal{W}_{u}(K ; E) .
\end{aligned}
$$

We are now in a position to prove Theorem 1.7.

Proof of Theorem 1.\%. On one hand, taking into account that the classical Wills functional does not depend on the dimension of the embedding space, a first upper bound is obtained from Proposition 5.1 applied to $f_{K, B_{2}^{n}}^{\pi(\cdot)^{2}}$ :

$$
\mathcal{W}\left(P_{H} K\right) \mathcal{W}\left(K \cap H^{\perp}\right) \leq\left(\begin{array}{l}
n \\
k
\end{array}\right) \mathcal{W}(K)
$$

On the other hand, since $\left\|f_{K, B_{2}^{n}}^{\pi(\cdot)^{2}}\right\|_{\infty}=f_{K, B_{2}^{n}}^{\pi(\cdot)^{2}}(0)$, using (2.10) we get

$$
(1-\lambda)^{k} \lambda^{n-k} \int_{H}\left(P_{H} f_{K, B_{2}^{n}}^{\pi(\cdot)^{2}}\right)(x)^{1-\lambda} \mathrm{d} x \int_{H^{\perp}} f_{K, B_{2}^{n}}^{\pi(\cdot)^{2}}(y)^{\lambda} \mathrm{d} y \leq \int_{\mathbb{R}^{n}} f_{K, B_{2}^{n}}^{\pi(\cdot)^{2}}(z) \mathrm{d} z .
$$

Now, since (cf. Lemma 2.3)

$$
\begin{aligned}
\left(P_{H} f_{K, B_{2}^{n}}^{\pi(\cdot)^{2}}\right)(x)^{1-\lambda} & =f_{P_{H} K, B_{2}^{k}}^{\pi(\cdot)^{2}}(x)^{1-\lambda}=e^{-(1-\lambda) \pi d\left(x, P_{H} K\right)^{2}} \\
& =e^{-\pi d\left(\sqrt{1-\lambda} x, \sqrt{1-\lambda} P_{H} K\right)^{2}}=f_{\sqrt{1-\lambda} P_{H} K, B_{2}^{k}}^{\pi(\cdot)^{2}}(\sqrt{1-\lambda} x)
\end{aligned}
$$


for every $x \in H$ and

$$
\begin{aligned}
f_{K, B_{2}^{n}}^{\pi(\cdot)^{2}}(y)^{\lambda} & \geq f_{K \cap H^{\perp}, B_{2}^{n-k}}^{\pi(\cdot)^{2}}(y)^{\lambda}=e^{-\lambda \pi d\left(y, K \cap H^{\perp}\right)^{2}} \\
& =e^{-\pi d\left(\sqrt{\lambda} y, \sqrt{\lambda} K \cap H^{\perp}\right)^{2}}=f_{\sqrt{\lambda} K \cap H^{\perp}, B_{2}^{n-k}}^{\pi(\cdot)^{2}}(\sqrt{\lambda} y)
\end{aligned}
$$

for all $y \in H^{\perp}$, doing the suitable change of variable in each integral we get

$$
\begin{aligned}
\frac{(1-\lambda)^{k} \lambda^{n-k}}{(1-\lambda)^{k / 2} \lambda^{(n-k) / 2}} & \int_{H} f_{\sqrt{1-\lambda} P_{H} K, B_{2}^{k}}^{\pi(\cdot)^{2}}(x) \mathrm{d} x \int_{H^{\perp}} f_{\sqrt{\lambda} K \cap H^{\perp}, B_{2}^{n-k}}^{\pi(\cdot)^{2}}(y) \mathrm{d} y \\
& \leq \int_{\mathbb{R}^{n}} f_{K, B_{2}^{n}}^{\pi(\cdot)^{2}}(z) \mathrm{d} z
\end{aligned}
$$

this is,

$$
(1-\lambda)^{k / 2} \lambda^{(n-k) / 2} \mathcal{W}\left(\sqrt{1-\lambda} P_{H} K\right) \mathcal{W}\left(\sqrt{\lambda} K \cap H^{\perp}\right) \leq \mathcal{W}(K) .
$$

Then, taking $\lambda=1 / 2$,

$$
\frac{1}{2^{n / 2}} \mathcal{W}\left(\frac{1}{\sqrt{2}} P_{H} K\right) \mathcal{W}\left(\frac{1}{\sqrt{2}} K \cap H^{\perp}\right) \leq \mathcal{W}(K)
$$

or equivalently,

$$
\mathcal{W}\left(P_{H} K\right) \mathcal{W}\left(K \cap H^{\perp}\right) \leq 2^{n / 2} \mathcal{W}(\sqrt{2} K)
$$

Together with (5.1) we get the result.

Remark 5.1. Since the maximum of the function $(1-\lambda)^{k / 2} \lambda^{(n-k) / 2}$ when $\lambda \in(0,1)$ is attained for $\lambda=(n-k) / n$, the best inequality which can be obtained from (5.2) would be

$$
\mathcal{W}\left(\left(\frac{k}{n}\right)^{1 / 2} P_{H} K\right) \mathcal{W}\left(\left(\frac{n-k}{n}\right)^{1 / 2} K \cap H^{\perp}\right) \leq \frac{n^{n / 2}}{k^{k / 2}(n-k)^{(n-k) / 2}} \mathcal{W}(K) .
$$

Remark 5.2. The minimum in Theorem 1.7 may be attained in both values, even for the same sets, depending on $k$. For instance, if we consider the unit cube $K=[0,1]^{n}$ and $E=B_{2}^{n}$, since $\mathrm{W}_{i}(K)=\kappa_{i}$ for $i=0, \ldots, n$, then

$$
\left(\begin{array}{l}
n \\
k
\end{array}\right) \mathcal{W}(K)=\left(\begin{array}{l}
n \\
k
\end{array}\right) 2^{n} \quad \text { and } \quad 2^{n / 2} \mathcal{W}(\sqrt{2} K)=\sum_{i=0}^{n}\left(\begin{array}{l}
n \\
i
\end{array}\right) 2^{n-i / 2}
$$

In dimension $n=10$, if $k=5$ then $2^{n / 2} \mathcal{W}(\sqrt{2} K)<\left(\begin{array}{l}n \\ k\end{array}\right) \mathcal{W}(K)$, whereas we get the opposite inequality when $k \neq 5$.

We conclude this subsection by showing some relations of the Wills functional of a convex body in terms of the Wills functional of certain projections of it onto hyperplanes. First, we recall some auxiliary results: if $f: \mathbb{R}^{n} \longrightarrow \mathbb{R}_{\geq 0}$ is a log-concave and integrable function, the polar projection body of $\bar{f}, \Pi^{*} f$, was introduced in [2], via the norm induced, by

$$
|v|_{\Pi^{*} f}=2 \int_{H_{v, 0}}\left(P_{H_{v}, 0} f\right)(x) \mathrm{d} x \quad \text { for all } v \in \mathbb{S}^{n-1} .
$$


Then, using polar coordinates, we have

$$
\operatorname{vol}\left(\Pi^{*} f\right)=\kappa_{n} \int_{\mathbb{S}^{n-1}} \frac{1}{|v|_{\Pi^{*} f}^{n}} \mathrm{~d} \sigma(v),
$$

where $\sigma$ denotes the Lebesgue probability measure on $\mathbb{S}^{n-1}$ (cf. [25, (1.53)]). Regarding a lower bound for $\operatorname{vol}\left(\Pi^{*} f\right)$, it was shown in $[2$, Theorem 1.1] that

$$
\int_{\mathbb{R}^{n}} \int_{\mathbb{R}^{n}} \min \{f(x), f(y)\} \mathrm{d} x \mathrm{~d} y \leq 2^{n} n !\|f\|_{\infty}\|f\|_{1}^{n+1} \operatorname{vol}\left(\Pi^{*} f\right) .
$$

Moreover, by the so-called affine Sobolev inequality (see [34, Theorem 1.1] and [2, page 2]), one has

$$
2^{n}\|f\|_{\frac{n}{n-1}}^{n} \operatorname{vol}\left(\Pi^{*} f\right) \kappa_{n-1}^{n} \leq \kappa_{n}^{n} .
$$

Using the above relations we can derive the maximal and minimal values of the Wills functional of the projections onto hyperplanes of a convex body, in terms of the Wills functional of the original set.

Theorem 5.1. Let $K \in \mathcal{K}^{n}$. Then

$$
\begin{aligned}
& \max _{v \in \mathbb{S}^{n-1}} \mathcal{W}\left(P_{H_{v, 0}} K\right) \geq 2 C_{n} \mathcal{W}(K)^{(n-1) / n} \quad \text { and } \\
& \min _{v \in \mathbb{S}^{n-1}} \mathcal{W}\left(P_{H_{v, 0}} K\right) \leq D_{n} \mathcal{W}(K)^{(n-1) / n}
\end{aligned}
$$

where $C_{n}$ and $D_{n}$ are given by

$$
C_{n}=\frac{1}{2}\left(\sqrt{\frac{n-1}{n}}\right)^{n-1} \frac{\kappa_{n-1}}{\kappa_{n}^{(n-1) / n}} \quad \text { and } \quad D_{n}=(n !)^{1 / n} \kappa_{n}^{1 / n} .
$$

Moreover,

$$
\sigma\left(v \in \mathbb{S}^{n-1}: \mathcal{W}\left(P_{H_{v, 0}} K\right) \geq C_{n} \mathcal{W}(K)^{(n-1) / n}\right) \geq 1-\frac{1}{2^{n}} .
$$

In particular, there exist absolute constants $C$ and $D$ such that

$$
\begin{aligned}
\max _{v \in \mathbb{S}^{n-1}} \mathcal{W}\left(P_{H_{v}, 0} K\right) & \geq C \mathcal{W}(K)^{(n-1) / n}, \\
\min _{v \in \mathbb{S}^{n-1}} \mathcal{W}\left(P_{H_{v, 0}} K\right) & \leq D \sqrt{n} \mathcal{W}(K)^{(n-1) / n} \quad \text { and } \\
\sigma\left(v \in \mathbb{S}^{n-1}: \mathcal{W}\left(P_{H_{v, 0}} K\right)\right. & \left.\geq C \mathcal{W}(K)^{(n-1) / n}\right) \geq 1-\frac{1}{2^{n}}
\end{aligned}
$$

for $n$ large enough.

Proof. For the sake of brevity we write $f=f_{K, B_{2}^{n}}^{\pi(\cdot)^{2}}$. On one hand, from (5.3) and Lemma 2.3 we obtain

$$
|v|_{\Pi^{*} f}=2 \mathcal{W}\left(P_{H_{v, 0}} K\right) .
$$

On the other hand, since $\|f\|_{\infty}=1$, then

$$
f(x) f(y) \leq \min \{f(x), f(y)\} \quad \text { for all } x, y \in \mathbb{R}^{n} .
$$


This fact, jointly with (5.5), implies that

$$
1 \leq 2^{n} n !\|f\|_{1}^{n-1} \operatorname{vol}\left(\Pi^{*} f\right)
$$

and hence, together with (5.6), we get

$$
\frac{1}{2(n !)^{1 / n}\|f\|_{1}^{(n-1) / n}} \leq \operatorname{vol}\left(\Pi^{*} f\right)^{1 / n} \leq \frac{\kappa_{n}}{2 \kappa_{n-1}\|f\|_{\frac{n}{n-1}}} .
$$

Taking into account (5.4), (2.14) and (5.9), the above inequality yields

$$
\begin{aligned}
\frac{1}{(n !)^{1 / n} \kappa_{n}^{1 / n} \mathcal{W}(K)^{(n-1) / n}} & \leq\left(\int_{\mathbb{S}^{n-1}} \frac{1}{\mathcal{W}\left(P_{H_{v}, 0} K\right)^{n}} \mathrm{~d} \sigma(v)\right)^{1 / n} \\
& \leq \frac{\kappa_{n}^{(n-1) / n}}{\kappa_{n-1}\left(\sqrt{\frac{n-1}{n}}\right)^{n-1} \mathcal{W}\left(\sqrt{\frac{n}{n-1}} K\right)^{(n-1) / n}},
\end{aligned}
$$

and then we infer that

$$
\max _{v \in \mathbb{S}^{n-1}} \mathcal{W}\left(P_{H_{v, 0}} K\right) \geq 2 C_{n} \mathcal{W}\left(\sqrt{\frac{n}{n-1}} K\right)^{(n-1) / n}
$$

and

$$
\min _{v \in \mathbb{S}^{n-1}} \mathcal{W}\left(P_{H_{v, 0}} K\right) \leq D_{n} \mathcal{W}(K)^{(n-1) / n}
$$

which imply (5.7), from the monotonicity (and the translation invariance) of the classical Wills functional.

To prove (5.8), we observe that (5.4) and Markov's inequality (see e.g. [10, Proposition 2.3.10]) imply that, for all $t>0$,

$$
\frac{1}{\kappa_{n}} \operatorname{vol}\left(\Pi^{*} f\right)=\int_{\mathbb{S}^{n-1}} \frac{1}{|v|_{\Pi^{*} f}^{n}} \mathrm{~d} \sigma(v) \geq \frac{1}{t^{n}} \sigma\left(v \in \mathbb{S}^{n-1}: \frac{1}{|v|_{\Pi^{*} f}^{n}} \geq \frac{1}{t^{n}}\right) .
$$

Then, taking

$$
s=\frac{t \kappa_{n}^{(n-1) / n}}{2\|f\|_{\frac{n}{n-1}} \kappa_{n-1}}
$$

and using (5.6), we get

$$
\sigma\left(v \in \mathbb{S}^{n-1}:|v|_{\Pi^{*} f} \leq \frac{2 s\|f\|_{\frac{n}{n-1}} \kappa_{n-1}}{\kappa_{n}^{(n-1) / n}}\right) \leq s^{n} .
$$

This inequality for $s=1 / 2$ jointly with (2.14) and (5.9) yield

$$
\sigma\left(v \in \mathbb{S}^{n-1}: \mathcal{W}\left(P_{H_{v}, 0} K\right) \leq C_{n} \mathcal{W}\left(\sqrt{\frac{n}{n-1}} K\right)^{(n-1) / n}\right) \leq \frac{1}{2^{n}},
$$

which implies (5.8), from the monotonicity (and the translation invariance) of the classical Wills functional. 
The last assertion follows from the fact that both $C_{n}$ and $D_{n} / \sqrt{n}$ are convergent to $1 / 2$ and $\sqrt{2 \pi / e}$, respectively, as may be seen by using Stirling's formula and the value of $\kappa_{n}$.

We note that Theorem 5.1 holds true in the general setting of the generalized Wills functional $\mathcal{W}_{u}(\cdot, E)$, but in that case, the bounds are given in terms of the functional $\mathcal{W}_{p u}(\cdot, E)$ for the suitable $p \geq 1$ (cf. (2.13)). We have settled the result for the classical functional because in this case the bounds are given in terms of $\mathcal{W}(\cdot)$ itself.

\subsection{Rogers-Shephard inequalities for the classical Wills functional.}

The classical Rogers-Shephard inequality for the difference body states that

$$
\operatorname{vol}(K-K) \leq\left(\begin{array}{c}
2 n \\
n
\end{array}\right) \operatorname{vol}(K)
$$

(see e.g. [25, Theorem 10.1.4]). A strengthening of this inequality was conjectured independently by Godbersen and Makai Jr., namely, that the mixed volume $\mathrm{V}(K[i],-K[n-i]) \leq\left(\begin{array}{c}n \\ i\end{array}\right) \operatorname{vol}(K)$ (see [25, Note 5 for Section 10.1] and the references therein). Engaging progresses have been made recently on this conjecture in [5]. Also the corresponding upper bounds for the intrinsic volumes $\mathrm{V}_{i}(K-K), i=1, \ldots, n-1$, are still unknown.

This subsection is devoted to studying Rogers-Shephard type inequalities for the classical Wills functional. We will provide two different upper bounds for $\mathcal{W}(K-K)$, which are obtained by using distinct techniques (we will exploit either the difference function, or a Rogers-Shephard type inequality for a log-concave function). These bounds will be not comparable in the sense that, depending on the dimension, one is better than the other.

First we prove Theorem 1.8. Indeed, profiting from (2.7), we get the following more general result for two convex bodies $K, L \in \mathcal{K}^{n}$.

Theorem 5.2. Let $K, L \in \mathcal{K}^{n}$ and let $\lambda \in(0,1)$. Then

$\mathcal{W}\left(\frac{(\lambda K) \cap((1-\lambda) L)}{\sqrt{\lambda(1-\lambda)}}\right) \mathcal{W}((1-\lambda) K-\lambda L) \leq \frac{1}{(\lambda(1-\lambda))^{n / 2}} \mathcal{W}(K) \mathcal{W}(L)$

When $\lambda=1 / 2$, we obtain Theorem 1.8 .

Proof of Theorem 5.2. Let $f=f_{K, B_{2}^{n}}^{\pi(\cdot)^{2}}((1-\lambda) \cdot)$ and $g=f_{L, B_{2}^{n}}^{\pi(\cdot)^{2}}(\lambda \cdot)$. Using Lemma 2.2 as well as the basic properties of the Asplund product we see that 
the $\lambda$-difference function associated to $f$ and $g$ can be written as

$$
\begin{aligned}
\Delta_{\lambda}^{f, g}(z) & =\sup _{z=x+y} f\left(\frac{x}{(1-\lambda)^{2}}\right)^{1-\lambda} g\left(\frac{-y}{\lambda^{2}}\right)^{\lambda} \\
& =\sup _{z=x+y} e^{-\pi(1-\lambda) d\left(\frac{x}{1-\lambda}, K\right)^{2}} e^{-\pi \lambda d\left(\frac{y}{\lambda},-L\right)^{2}} \\
& =\sup _{z=x+y} e^{-\frac{\pi}{1-\lambda} d(x,(1-\lambda) K)^{2}} e^{-\frac{\pi}{\lambda} d(y,-\lambda L)^{2}} \\
& =\left(\left(e^{-\frac{\pi}{1-\lambda}|\cdot|^{2}} \star \chi_{(1-\lambda) K}\right) \star\left(e^{-\frac{\pi}{\lambda}|\cdot|^{2}} \star \chi_{-\lambda L}\right)\right)(z) \\
& =\left(e^{-\frac{\pi}{1-\lambda}|\cdot|^{2}} \star e^{-\frac{\pi}{\lambda}|\cdot|^{2}} \star \chi_{(1-\lambda) K-\lambda L}\right)(z) .
\end{aligned}
$$

Since, for any $v \in \mathbb{R}^{n}$,

$$
\begin{aligned}
\left(e^{\left.-\frac{\pi}{1-\lambda}|\cdot|^{2} \star e^{-\frac{\pi}{\lambda}|\cdot|^{2}}\right)(v)}\right. & =\sup _{w \in \mathbb{R}^{n}} e^{-\pi\left(\frac{|w|^{2}}{1-\lambda}+\frac{|v-w|^{2}}{\lambda}\right)} \\
& =e^{-\frac{\pi}{\lambda(1-\lambda)} \inf _{w \in \mathbb{R}^{n}}\left(\lambda|w|^{2}+(1-\lambda)|v-w|^{2}\right)} \\
& =e^{-\frac{\pi}{\lambda(1-\lambda)} \inf _{w \in \mathbb{R}^{n}}\left(|w|^{2}+(1-\lambda)|v|^{2}-2(1-\lambda)\langle v, w\rangle\right)} \\
& =e^{-\frac{\pi}{\lambda(1-\lambda)} \inf _{r \geq 0} \inf _{|w|=r|v|}\left(r^{2}|v|^{2}+(1-\lambda)|v|^{2}-2(1-\lambda)\langle v, w\rangle\right)} \\
& =e^{-\frac{\pi}{\lambda(1-\lambda)} \inf _{r \geq 0}\left(r^{2}+1-\lambda-2 r(1-\lambda)\right)|v|^{2}} \\
& =e^{-\frac{\pi}{\lambda(1-\lambda)}\left(1-\lambda-(1-\lambda)^{2}\right)|v|^{2}}=e^{-\pi|v|^{2}},
\end{aligned}
$$

then (5.10) yields $\Delta_{\lambda}^{f, g}=e^{-\pi|\cdot|^{2}} \star \chi_{(1-\lambda) K-\lambda L}$. Thus, by Lemma $2.2, \Delta_{\lambda}^{f, g}=$ $f_{(1-\lambda) K-\lambda L, B_{2}^{n}}^{\pi(\cdot)^{2}}$ and hence

$$
\int_{\mathbb{R}^{n}} \Delta_{\lambda}^{f, g}(x) \mathrm{d} x=\mathcal{W}((1-\lambda) K-\lambda L) .
$$

Moreover, we clearly have that

$$
\begin{aligned}
f(x)^{\lambda} g(x)^{1-\lambda} & =e^{-\pi \lambda d((1-\lambda) x, K)^{2}} e^{-\pi(1-\lambda) d(\lambda x, L)^{2}} \\
& =e^{-\pi \lambda(1-\lambda)^{2} d\left(x, \frac{K}{1-\lambda}\right)^{2}} e^{-\pi(1-\lambda) \lambda^{2} d\left(x, \frac{L}{\lambda}\right)^{2}} \\
& \geq e^{-\pi \lambda(1-\lambda)^{2} d\left(x, \frac{K}{1-\lambda} \cap \frac{L}{\lambda}\right)^{2}} e^{-\pi(1-\lambda) \lambda^{2} d\left(x, \frac{K}{1-\lambda} \cap \frac{L}{\lambda}\right)^{2}} \\
& =e^{-\pi d\left(\sqrt{\lambda(1-\lambda)} x, \frac{(\lambda K) \cap((1-\lambda) L)}{\sqrt{\lambda(1-\lambda)}}\right)^{2}},
\end{aligned}
$$

and then, applying the change of variable $y=\sqrt{\lambda(1-\lambda)} x$, we get

$$
\int_{\mathbb{R}^{n}} f(x)^{\lambda} g(x)^{1-\lambda} \mathrm{d} x \geq \frac{1}{(\lambda(1-\lambda))^{n / 2}} \mathcal{W}\left(\frac{(\lambda K) \cap((1-\lambda) L)}{\sqrt{\lambda(1-\lambda)}}\right) .
$$


Therefore, by (2.7) and doing the changes of variable $y=(1-\lambda) x$ and $y=\lambda x$, respectively, we obtain

$$
\begin{aligned}
& \mathcal{W}\left(\frac{(\lambda K) \cap((1-\lambda) L)}{\sqrt{\lambda(1-\lambda)}}\right) \mathcal{W}((1-\lambda) K-\lambda L) \\
& \leq(\lambda(1-\lambda))^{n / 2} \int_{\mathbb{R}^{n}} f(x) \mathrm{d} x \int_{\mathbb{R}^{n}} g(y) \mathrm{d} y=\frac{1}{(\lambda(1-\lambda))^{n / 2}} \mathcal{W}(K) \mathcal{W}(L)
\end{aligned}
$$

This concludes the proof.

It is known (see [4, Theorem 2.2] as well as [11] for related inequalities) that for a log-concave function $f: \mathbb{R}^{n} \longrightarrow \mathbb{R}_{\geq 0}$,

$$
\int_{\mathbb{R}^{n}}(f \star \bar{f})(x) \mathrm{d} x \leq\left(\begin{array}{c}
2 n \\
n
\end{array}\right)\|f\|_{\infty} \int_{\mathbb{R}^{n}} f(x) \mathrm{d} x,
$$

where $\bar{f}: \mathbb{R}^{n} \longrightarrow \mathbb{R}_{\geq 0}$ is given by $\bar{f}(x)=f(-x)$.

We conclude the paper by using this result to obtain the last announced upper bound for the classical Wills functional of the difference body.

Theorem 5.3. Let $K \in \mathcal{K}^{n}$. Then

$$
\mathcal{W}(K-K) \leq \frac{\left(\begin{array}{c}
2 n \\
n
\end{array}\right)}{2^{n / 2}} \mathcal{W}(\sqrt{2} K) .
$$

Proof. Using (5.11) for $\lambda=1 / 2$, namely $e^{-2 \pi|\cdot|^{2}} \star e^{-2 \pi|\cdot|^{2}}=e^{-\pi|\cdot|^{2}}$, we obtain that $e^{-\pi|\cdot|^{2}} \star e^{-\pi|\cdot|^{2}}=e^{-\frac{\pi}{2}|\cdot|^{2}}$ and thus, by Lemma 2.2,

$f_{K, B_{2}^{n}}^{\pi(\cdot)^{2}} \bar{f}_{K, B_{2}^{n}}^{\pi(\cdot)^{2}}=\left(e^{-\pi|\cdot|^{2}} \star \chi_{K}\right) \star\left(e^{-\pi|\cdot|^{2}} \star \chi_{-K}\right)=e^{-\frac{\pi}{2}|\cdot|^{2} \star \chi_{K-K}}=f_{K-K, B_{2}^{n}}^{\pi(\cdot)^{2} / 2}$.

Since

$$
\begin{aligned}
\int_{\mathbb{R}^{n}} f_{K-K, B_{2}^{n}}^{\pi(\cdot)^{2} / 2}(x) \mathrm{d} x & =\int_{\mathbb{R}^{n}} e^{-\frac{\pi}{2} d(x, K-K)^{2}} \mathrm{~d} x=\int_{\mathbb{R}^{n}} e^{-\pi d\left(\frac{x}{\sqrt{2}}, \frac{K-K}{\sqrt{2}}\right)^{2}} \mathrm{~d} x \\
& =2^{n / 2} \mathcal{W}\left(\frac{K-K}{\sqrt{2}}\right),
\end{aligned}
$$

then (5.12) yields

$$
\mathcal{W}\left(\frac{K-K}{\sqrt{2}}\right) \leq \frac{\left(\begin{array}{c}
2 n \\
n
\end{array}\right)}{2^{n / 2}} \mathcal{W}(K),
$$

which concludes the proof.

Remark 5.3. We observe that the bounds (1.6) and (5.13) are not comparable. For instance, if we consider the cube $K=[0,1 / 2]^{n}$, for which $\mathrm{W}_{i}(K)=\kappa_{i} / 2^{n-i}, i=0, \ldots, n$, then it is easy to check that

$$
2^{n} \mathcal{W}(2 K)=4^{n}<\left(\begin{array}{c}
2 n \\
n
\end{array}\right) \sum_{i=0}^{n}\left(\begin{array}{c}
n \\
i
\end{array}\right) \frac{1}{2^{(n+i) / 2}}=\frac{\left(\begin{array}{c}
2 n \\
n
\end{array}\right)}{2^{n / 2}} \mathcal{W}(\sqrt{2} K)
$$

for $n=9$, whereas we get the opposite inequality when $n=3$. 


\section{REFERENCES}

[1] D. Alonso-Gutiérrez, S. Artstein-Avidan, B. González, C. H. Jiménez, R. Villa, Rogers-Shephard and local Loomis-Whitney type inequalities, To appear in Math. Ann., https://doi.org/10.1007/s00208-019-01834-3.

[2] D. Alonso-Gutiérrez, J. Bernués, B. González, Zhang's inequality for log-concave functions, To appear in GAFA Seminar Notes.

[3] D. Alonso-Gutiérrez, J. Bernués, B. González, An extension of Berwald's inequality and its relation to Zhang's inequality, Preprint, arXiv:1908.01154.

[4] D. Alonso-Gutiérrez, B. González, C. H. Jiménez, R. Villa, Rogers-Shephard inequality for log-concave functions, J. Funct. Anal. 271 (11) (2016), 3269-3299.

[5] S. Artstein-Avidan, K. Einhorn, D. I. Florentin, Y. Ostrover, On Godbersen's conjecture, Geom. Dedicata 178 (2015), 337-350.

[6] S. Artstein-Avidan, B. Klartag, V. Milman, The Santaló point of a function, and a functional form of the Santaló inequality, Mathematika 51 (1-2) (2014), 33-48.

[7] K. Ball, Volumes of sections of cubes and related problems, Geometric Aspects of Functional Analysis, Lecture Notes in Math. 1376, 251-260, Springer, Berlin, 1989.

[8] L. Berwald: Verallgemeinerung eines Mittelwertsatzes von J. Favard für positive konkave Funktionen, Acta Math. 79 (1947), 17-37.

[9] U. Betke, M. Henk, Intrinsic volumes and lattice points of crosspolytopes, Monatsh. Math. 115 (1-2) (1993), 27-33.

[10] D. L. Cohn, Measure theory, 2nd revised ed., Birkhäuser/Springer, New York Heidelberg, 2013.

[11] A. Colesanti, Functional inequalities related to the Rogers-Shephard inequality, Mathematika 53 (2006), 81-101.

[12] A. Colesanti, E. Saorín Gómez, J. Yepes Nicolás, On a linear refinement of the Prékopa-Leindler inequality, Canad. J. Math. 68 (4) (2016), 762-783.

[13] R. J. Gardner, The Brunn-Minkowski inequality, Bull. Amer. Math. Soc. 39 (3) (2002), 355-405.

[14] P. M. Gruber, Convex and Discrete Geometry, Springer, Berlin Heidelberg, 2007.

[15] H. Hadwiger, Das Wills'sche Funktional, Monatsh. Math. 79 (1975), 213-221.

[16] H. Hadwiger, Gitterpunktanzahl im Simplex und Wills'sche Vermutung, Math. Ann. 239 (3) (1979), 271-288.

[17] M. A. Hernández Cifre, J. Yepes Nicolás, On the roots of the Wills functional, J. Math. Anal. Appl. 401 (2013), 733-742.

[18] M. A. Hernández Cifre, J. Yepes Nicolás, On the roots of generalized Wills $\mu$ polynomials, Rev. Mat. Iberoamericana 31 (2) (2015), 477-496.

[19] J. Kampf, On weighted parallel volumes, Beiträge Algebra Geom. 50 (2) (2009), 495-519.

[20] B. Klartag, V. D. Milman, Geometry of log-concave functions and measures, Geom. Dedicata 112 (2005), 169-182.

[21] P. McMullen, Non-linear angle-sum relations for polyhedral cones and polytopes, Math. Proc. Cambridge Philos. Soc. 78 (1975), 247-261.

[22] P. McMullen, Inequalities between intrinsic volumes, Monatsh. Math. 111 (1) (1991), 47-53.

[23] V. D. Milman, Inégalité de Brunn-Minkowski inverse et applications à la théorie locale des espaces normés (An inverse form of the Brunn-Minkowski inequality, with applications to the local theory of normed spaces), C. R. Acad. Sci. Paris Ser. I Math. 302 (1) (1986), 25-28.

[24] J. R. Sangwine-Yager, Mixed volumes. In: Handbook of Convex Geometry (P. M. Gruber and J. M. Wills eds.), North-Holland, Amsterdam, 1993, 43-71. 
[25] R. Schneider, Convex bodies: The Brunn-Minkowski theory, 2nd expanded ed. Encyclopedia of Mathematics and its Applications 151, Cambridge, Cambridge University Press, 2014.

[26] R. A. Vitale, The Wills functional and Gaussian processes, Ann. Probab. 24 (4) (1996), 2172-2178.

[27] R. A. Vitale, A log-concavity proof for a Gaussian exponential bound, Contemp. Math. 239 (1999), 209-212.

[28] R. A. Vitale, Intrinsic volumes and Gaussian processes, Adv. Appl. Prob. (SGSA) 33 (2001), 354-364.

[29] R. A. Vitale, Y. Wang, The Wills functional for Poisson processes, Statist. Probab. Lett. 78 (14) (2008), 2181-2187.

[30] R. A. Vitale, On an exponential functional for Gaussian processes and its geometric foundations, J. Math. Sci. 238 (4) (2019), 406-414.

[31] J. M. Wills, Zur Gitterpunktanzahl konvexer Mengen, Elem. Math. 28 (1973), 57-63.

[32] J. M. Wills, Nullstellenverteilung zweier konvexgeometrischer Polynome, Beiträge Algebra Geom. 29 (1989), 51-59.

[33] J. M. Wills, Minkowski's successive minima and the zeros of a convexity-function, Monatsh. Math. 109 (2) (1990), 157-164.

[34] G. Zhang, The affine Sobolev inequality, J. Differ. Geom. 53 (1) (1999), 183-202.

Departamento de Matemáticas, Universidad de Zaragoza, C/ Pedro CerBuna 12, 50009-ZARAgOzA, Spain

E-mail address: alonsod@unizar.es

Departamento de Matemáticas, Universidad de Murcia, Campus de EspinarDO, 30100-Murcia, SPAin

E-mail address: mhcifre@um.es

E-mail address: jesus.yepes@um.es 